\title{
Article
}

\section{Protein abundance and folding rather than the redox state of Kelch13 determine the artemisinin susceptibility of Plasmodium falciparum}

Robin Schumann', Eileen Bischoff', Severina Klaus², Sophie Möhring', Julia Flock ${ }^{3}$, Sandro Keller ${ }^{4-7}$, Kim Remans ${ }^{3}$, Markus Ganter ${ }^{2}$ \& Marcel Deponte $^{1 \star}$

${ }^{1}$ Faculty of Chemistry, Comparative Biochemistry, Technische Universität Kaiserslautern, D67663 Kaiserslautern, Germany

${ }^{2}$ Centre for Infectious Diseases, Parasitology, Heidelberg University Hospital, D-69120 Heidelberg, Germany

${ }^{3}$ Protein Expression and Purification Core Facility, European Molecular Biology Laboratory (EMBL), D-69117 Heidelberg, Germany

${ }^{4}$ Molecular Biophysics, Technische Universität Kaiserslautern, D-67663 Kaiserslautern, Germany

${ }^{5}$ Biophysics, Institute of Molecular Biosciences (IMB), NAWI Graz, University of Graz, Humboldtstr. 50/III, 8010 Graz, Austria

${ }^{6}$ Field of Excellence BioHealth, University of Graz, Graz, Austria

${ }^{7}$ BioTechMed-Graz, Graz, Austria

${ }^{*}$ Correspondence and requests for materials should be addressed to M.D. (deponte@chemie.uni-kl.de) 


\section{Abstract}

Decreased susceptibilities of Plasmodium falciparum towards the endoperoxide antimalarial artemisinin are linked to mutations of residue C580 of Kelch13, which is the homologue of the redox sensor Keap1 in vertebrates. Here, we addressed whether mutations alter the artemisinin susceptibility by modifying the redox properties of Kelch13 or by compromising its native fold or abundance. Using selection-linked integration and the $g / m S$ ribozyme, efficient down-regulation of Kelch13 resulted in ring-stage survival rates around $40 \%$. While the loss of a potential disulfide bond between residues C580 and C532 had no effect on the artemisinin suceptibility, the thiol group of $\mathrm{C} 473$ could not be replaced. We also established a protocol for the production of recombinant Kelch13. In contrast to cysteine-to-serine replacements, common field mutations resulted in misfolded and insoluble protein. In summary, not the redox properties but impaired folding of Kelch13, resulting in a decreased Kelch13 abundance, is the central parameter for mutant selection. 


\section{Introduction}

Artemisinin and its derivates are central to the treatment of malaria and are recommended as first-line drugs for artemisinin-based combination therapies by the World Health Organization'. In 2009, Dondorp et al. reported a delayed clearance of the malaria parasite Plasmodium falciparum in patients following artesunate treatment ${ }^{2}$. The delayed parasite clearance was found to be associated with reduced drug susceptibility of the ring stage $\mathrm{e}^{3-6}$ as well as mutations in PFKELCH13, which encodes a kelch domain-containing protein on chromosome $13^{7-9}$. PfKelch13 belongs to the top $5 \%$ of most conserved proteins in Plasmodium and comprises an N-terminal apicomplexan-specific region followed by a CCC domain, a BTB domain, and a six bladed kelch $\beta$-propeller domain ${ }^{10}$. Relevant mutations for delayed parasite clearance were predominantly found in the $\beta$-propeller domain of PfKelch13, with C580Y being the most prevalent one $\mathrm{e}^{7,8}$. Mutations R539T or I543T are less frequent but were reportd to lead to even higher ring-stage survival in vitro ${ }^{9}$. While decreased artemisinin susceptibilities were initially restricted to hot spots at the Thai-Cambodian border, non-related C580Y mutant strains have recently been detected in South America ${ }^{11}$ and on New Guinea ${ }^{12}$. Furthermore, a novel strain with a R561H mutation has emerged in Rwuanda ${ }^{13}$. Hence, PfKelch13 mutations endanger the long-term goal to eliminate malaria ${ }^{14}$.

N-terminally GFP-tagged wild-type and C580Y mutant PfKelch13 localize to the same punctate structures close to the digestive vacuole ${ }^{15,16}$ and were found in ring-shaped cytostome-like structures at the plasma membrane ${ }^{17}$. Furthermore, tagged and untagged wildtype and mutant PfKelch13 variants were reported to localize to cytosolic foci, the endoplasmic reticulum, vesicular structures, and the mitochondrion ${ }^{18,19}$. Mislocalization and hemoglobin uptake studies in combination with a dimerization-induced quantitative BiolD PfKelch13 interactome revealed an involvment of PfKelch13 in endocytosis, suggesting that PfKelch13 mutations result in a decreased protein abundance, hemoglobin uptake, and activation of artemisinin ${ }^{16,17}$. This theory has been supported so far by mislocalization and overexpression studies using protein-tagged wild-type or mutant PfKelch13 ${ }^{16-18}$. 
PfKelch13 is highly similar to Keap $1^{8,10}$, which is the master redox and electrophile sensor in mammals and which interacts with the transcription factor Nrf2 via its $\beta$-propeller domain ${ }^{20,21}$. Keap1-bound Nrf2 becomes ubiquitinated and undergoes proteasomal degradation in the cytsosol $^{22,23}$. Oxidation or alkylation alters the conformation of Keap1, resulting in the liberation and translocation of Nrf2 to the nucleus ${ }^{20-24}$. Nuclear Nrf2 forms heterodimers and binds with its basic leucine zipper domain to the electrophile-responsive element (EpRE), resulting in a plethora of adaptive responses such as the induction of phase II detoxifying enzymes and the synthesis of glutathione ${ }^{21,25,26}$. Although $P$. falciparum blood stages are thought to adapt to numerous endogenous and environmental oxidative challenges, they lack an Nrf2 homologue ${ }^{8}$. Whether PfKelch13 also acts as a redox sensor (e.g., based on residue C580), and whether the endoperoxide artemisinin interferes with such a function, remained to be studied.

Here, we used selection-linked integration $(S L I)^{15}$ in combination with g/mS ribozymetagging $^{27}$ and established a purification protocol for recombinant PfKelch13 to study the relevance of the abundance, conformational stability, and redox state of PfKelch13 for the artemisinin susceptibility in $P$. falciparum.

\section{Results}

\section{Knockdown of PFKELCH13 decreases parasite growth}

To test the relevance of the PfKelch13 abundance on the growth of $P$. falciparum blood stages, we used the SLI method by Birnbaum et al. ${ }^{15}$ to generate a 3D7 strain with Hiss-tagged PfKelch13 that is under control of the glmS ribozyme from Bacillus subtilis ${ }^{27}$ (Fig. 1a,b). The ribozyme can be activated by the addition of glucosamine (GlcN), resulting in the degradation of the His 8 -PfKelch13-encoding mRNA. A strain with the inactive mutant $g / m S M 9$ was generated in parallel and served as a negative control. Following successful selection with the P. falciparum dihydroorotate dehydrogenase inhibitor DSM1, plasmid integration and 
disruption of endogenous PFKELCH13 was confirmed by analytical PCR for both strains (Fig. 1c). Furthermore, PCR analyses with a primer pair that binds to endogenous PFKELCH13 upand downstream of the homology region (and that does not recognize recodonized His - $^{-}$ PFKELCH13) confirmed the loss of the original PFKELCH13 copy in the whole genome. The effect of GlcN was tested for asynchronous blood-stage cultures of strains His 8 -PFKELCH13glmS and His ${ }_{8}$-PFKELCH13-M9 (Fig. 1d). After five days, strain His ${ }_{8}-$ PFKELCH13-glmS grew normal in the presence of $0.3 \mathrm{mM} \mathrm{GlcN}$, whereas treatments with $1.0 \mathrm{mM}$ and $2.0 \mathrm{mM}$ GlcN resulted in growth inhibitions around $25 \%$ and more than $50 \%$, respectively. To confirm a genespecific knockdown effect and to exclude a growth inhibitory effect of GlcN, we treated $\mathrm{His}_{8}$ PFKELCH13-M9 with the same GlcN concentrations. While 0.3 and $1.0 \mathrm{mM}$ GlcN had no effect on parasite growth, a growth inhibition around $30 \%$ was detected for the negative control after five days of treatment with $2.0 \mathrm{mM}$ GlcN. Complete growth arrest was observed for both strains in the presence of 5 and $10 \mathrm{mM}$ GlcN, further supporting the reported toxicity of higher GlcN concentrations $^{28}$. In summary, we established that up to $2.0 \mathrm{mM}$ GlcN can be used for knockdown studies with $\mathrm{His}_{8}-$ PFKELCH13-glmS and that the addition of $1.0 \mathrm{mM} \mathrm{GlcN}$ resulted in a specific growth defect because of the down-regulation of His 8 -PfKelch13.

\section{Quantification of the down-regulation of His ${ }_{8}$-PfKelch13}

The resistance marker yeast dihydroorotate dehydrogenase $(\mathrm{yDHODH})$ and His 8 -PfKelch13 are encoded by the same mRNA (Fig. 1a). We therefore used the DSM1 resistance of strains $\mathrm{His}_{8}$-PFKELCH13-gImS and His ${ }_{8}$-PFKELCH13-M9 as a quantitative readout for the glmSdependent down-regulation of His - PfKelch13 (Fig. 2a). The parental 3D7 strain served as a

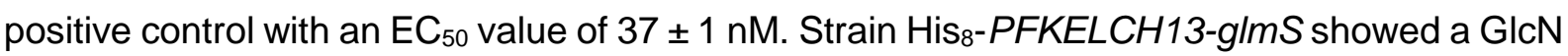
concentration-dependent loss of resistance towards DSM1. While $0.3 \mathrm{mM} \mathrm{GlcN}$ only slightly increased the DSM1 susceptibility, treatments with 1.0 and $2.0 \mathrm{mM}$ GlcN resulted in $\mathrm{EC}_{50}$ values of $350 \pm 30$ and $116 \pm 8 \mathrm{nM}$, respectively. Strain His - PFKELCH13-M9 remained fully resistant even in the presence of $2.0 \mathrm{mM} \mathrm{GlcN}$. Thus, the loss of resistance against DSM1 
confirmed the efficient gImS-dependent knockdown of the mRNA fusion construct between yDHODH and His ${ }_{8}-$ PFKELCH13.

Next, we performed western blot analyses against the His-tag to quantify the efficiency of the down-regulation of His8-PfKelch13. Four independent experiments showed a strong GlcN concentration-dependent down-regulation of Hiss-PfKelch13 (Fig. 2b). Since the antibody detected two regulated bands in parasite lysates (as outlined below), we quantified the intensity as the sum of both bands and normalized it against western blot signals for aldoalase as a housekeeping protein. In the presence of $\mathrm{GlcN}$, His ${ }_{8}$-PfKelch13 levels decreased in a concentration-dependent manner. The addition of $1.0 \mathrm{mM} \mathrm{GlcN}$ resulted in a down-regulation of $65 \pm 10 \%$, whereas $2.0 \mathrm{mM}$ GlcN resulted in a down-regulated by $93 \pm 2 \%$ (Fig. $2 \mathrm{~b}, \mathrm{c}$ ). Treatment of strain His8-PFKELCH13-M9 with the same GIcN concentrations did not reveal significantly lower His -PfKelch13 levels. In summary, the glmS system allowed us to titrate the level of His 8 -PfKelch13 with GlcN and to down-regulate the protein by more than $90 \%$.

\section{Down-regulation of His 8 -PfKelch13 decreases artemisinin susceptibility}

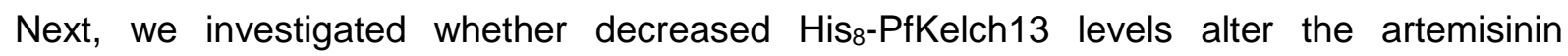
susceptibility comparable to PfKelch13 mutants in the field. Pretreatment of strain His8PFKELCH13-glmS with $1.0 \mathrm{mM} \mathrm{GlcN}$ followed by a ring-stage survival assay (RSA) with artesunate without GlcN resulted in survival rates of more than $35 \%$ (Fig. 3a). Increasing the GlcN concentration during the preincubation to $2.0 \mathrm{mM}$ slightly improved the survival rate, suggesting a survival rate limit around $40 \%$, a value more than twice as high as the survival rate for the positive control NF54K13 $3^{\mathrm{C} 580 \mathrm{Y}}$ at around $14 \%$. The negative control $\mathrm{His}_{8}{ }^{-}$ PFKELCH13-M9 did not show significantly increased survival rates in the presence of GlcN. In summary, down-regulation of His - PfKelch13 led to a decreased artemisinin susceptibility with a striking survival rate slightly above $40 \%$. 


\section{Down-regulation of His8-PfKelch13 impairs hemozoin formation}

Parasites with a decreased artemisinin susceptibility as well as parasites with mislocalized PfKelch13 were shown to remain in a prolonged ring stage with a decreased endocytotic activity ${ }^{15,29,30}$. We therefore analyzed whether down-regulation of His 8 -PfKelch13 affects the formation of hemozoin from endocytosed hemoglobin using synchronized parasite cultures (Fig. 3b, Fig. S1). A phenotype in the presence of $1 \mathrm{mM}$ GlcN was detected by light microscopy during the second intraerythrocytic cycle. While hemozoin was visible in all parasites from strains His ${ }_{8}-$ PFKELCH13-gImS and His ${ }^{-}$PFKELCH13-M9 around $28 \mathrm{~h}$ post-invasion (hpi) during the first cycle, only around $30 \%$ of $\mathrm{His}_{8}$-PFKELCH13-gImS parasites contained visible amounts of hemozoin 28-32 hpi during the second cycle. Thus, down-regulation of $\mathrm{His}_{8}{ }^{-}$ PfKelch13 phenocopied the delayed development and impaired hemozoin formation of strains with mutant PfKelch13 variants.

\section{Relevance of the thiol groups of C469, C473, C532, and C580}

Next, we addressed whether PfKelch13 has a Keap1-like redox function and whether such a function might play a role for the artemisinin susceptibility. First, we determined the $\mathrm{IC}_{50}$ values of the disulfide-inducer diamide, the oxidant tert-butyl hydroperoxide $(\mathrm{tBuOOH})$, the endoperoxide artesunate, and the reductant dithiothreitol (DTT) for strain NF54K13 ${ }^{\mathrm{C} 580 \mathrm{Y}}$ from Ghorbal et al. ${ }^{31}$ (Fig. S2). Strain NF54 served as a control. The $\mathrm{IC}_{50}$ values were almost identical for both strains and were in good agreement with previous measurements for strain $3 \mathrm{D} 7^{32,33}$.

PfKelch13 has seven cysteine residues, all of which are within the kelch $\beta$-propeller domain (Fig. 4a). Residues C532 and C580 can form a disulfide bond between two blades at the center of the $\beta$-propeller (Fig. 4b). Free access to residue C532 from the top site of the $\beta$-propeller is partially blocked by residues $\mathrm{Y} 480$ and N530, whereas smaller molecules might access the sulfur atom of residue C580 from the bottom site next to the BTB domain. To test the physiological relevance of the redox state of residues C532 and C580, we used SLI to generate mutant strains $\mathrm{His}_{8}-$ PFKELCH13 ${ }^{\mathrm{C532S}}$ and $\mathrm{His}_{8}-$ PFKELCH13 ${ }^{\mathrm{C580S}}$ (Fig. S3a). Strain His $8^{-}$ 
PFKELCH13 ${ }^{\text {wt }}$ with His-tagged wild-type PfKelch13 was generated as a control. PCR analysis confirmed the plasmid integration and disruption of endogenous PFKELCH13 for all three strains (Fig. S3b). RSAs with artesunate did not reveal an altered artemisinin susceptibility for strains His ${ }_{8}-P F K E L C H 13^{C 532 S}$ and $\mathrm{His}_{8}-P F K E L C H 13^{C 580 S}$ (Fig. 4c). Strain NF54K13 ${ }^{\mathrm{C} 580 \mathrm{Y}}$ served again as a positive control.

In contrast to previous reports on the generation of PFKELCH13 ${ }^{C 580 Y}$ mutants ${ }^{15,31}$, we were unable to generate strain $\mathrm{His}_{8}-P F K E L C H 13^{C 580 Y}$. Two out of three unsuccessful SLI

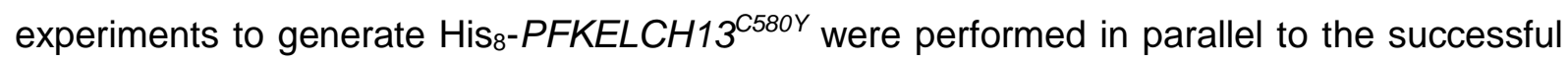
generation of other $\mathrm{His}_{8}-$ PFKELCH13 mutants, suggesting that the C580Y mutation in combination with the $\mathrm{His}_{8}$-tag and altered codon usage might be lethal. We also tried to replace the only two surface-exposed cysteine residues C469 and C473 (Fig. 4a,b). While we were able to readily generate strain $\mathrm{His}_{8}-P F K E L C H 13^{C 469 S}$ (Fig. S3b), three SLI attempts to generate His ${ }_{8}-P F K E L C H 13^{C 473 S}$ were unsuccessful (two of which were again performed in parallel to successful SLI experiments). Taking into account that only a single surface-exposed oxygen atom is replaced in $\mathrm{His}_{8}-$ PFKELCH13 ${ }^{\mathrm{C} 473 S}$, we speculate that the thiol group of residue $\mathrm{C} 473$

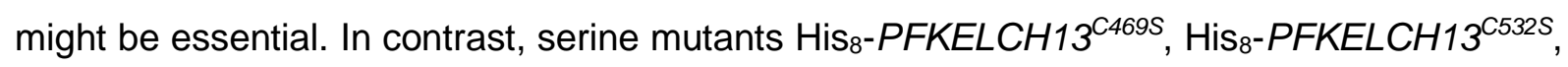
and $\mathrm{HiS}_{8}-$ PFKELCH13 ${ }^{\mathrm{C580S}}$ showed no growth defect (Fig. 4d).

In summary, strain NF54K13 ${ }^{\mathrm{C} 580 \mathrm{Y}}$ has a normal susceptibility to common redox agents. Not the loss of the thiol group of C580 but the specific replacement by tyrosine increases the artemisinin susceptibility of $P$. falciparum. However, a Keap1-like redox function of PfKelch13 cannot be excluded at the current stage because of three unsuccessful attempts to replace the thiol group of surface-exposed residue C473.

\section{Characterization of recombinant PfKelch13 and antibody generation}

To the best of our knowledge, no protocol for the production of recombinant PfKelch13 has been published yet despite the deposition of PDB entries 4ZGC and 4YY8 by Jiang et al. and the Structural Genomics Consortium (SGC) (DOI: 10.2210/pdb4zgc/pdb). To study the effects of common mutations as well as the protein properties of PfKelch13 in more detail, we 
produced recombinant full-length PfKelch13 as well as truncated PfKelch13 $337-726$ that comprises the BTB domain and the Kelch $\beta$-propeller domain (Fig. 5a). Recombinant fulllength PfKelch or PfKelch13 ${ }^{337-726}$ produced in Escherichia coli was insoluble despite numerous parameter variations (including alternative strains, temperatures, IPTG- or autoinduction, N-terminal $\mathrm{His}_{6}{ }^{-}$, GST- or MBP-tags or the systematic replacement of cysteine residues), indicating intrinsic protein folding problems most likely because of the $\beta$-propeller

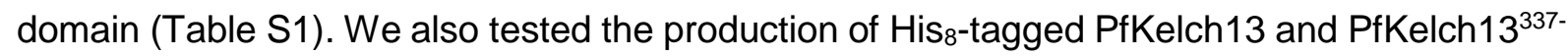
726 in Pichia pastoris GS115 and the Leishmania tarentolae-based LEXSY system but did not obtain soluble recombinant protein form these eukaryotes. Nevertheless, we established a solubilization and purification protocol for recombinant $\mathrm{MAH}_{6}$ VGT-tagged PfKelch13 ${ }^{336-726}$ from inclusion bodies in E. coli to generate and affinity-purify an antibody against PfKelch13 (Fig. S4a-c). Western blot analyses using this antibody revealed a specific detection of endogenous PfKelch13 at $\sim 85 \mathrm{kDa}$ in $P$. falciparum lysates. Furthermore, we coupled the antibody to $\mathrm{CNBr}$ activated sepharose and pulled-down denatured PfKelch13 and His s $_{8}$-PfKelch13 ${ }^{\text {wt }}$ from parasite lysates of strains $3 \mathrm{D} 7$ and $\mathrm{His}_{8}-P F K E L C H 13^{\text {wt }}$, respectively (Fig. S4d). The eluate containing His ${ }_{8}$-PfKelch13 $1{ }^{\text {wt }}$ was probed with an antibody against the His-tag (Fig. S4d) and was also analyzed by mass spectrometry (as outlined below), confirming the specifity of the antibody against PfKelch13 and the successful pull-down. Since disulfide bonds are maintained under denaturing conditions, the established protocol can be applied in future studies to identify potential redox interaction partners using wild-type PfKelch13 and cysteine mutants.

Next, we produced and purified recombinant $\mathrm{MH}_{8}$ SR-tagged PfKelch13337-726 from Spodoptera frugiperda Sf21 cells (Fig. S5a). The yield was around $6 \mathrm{mg}$ of pure soluble protein per liter of culture. SDS-PAGE analysis showed an apparent molecular mass of $43 \mathrm{kDa}$ in accordance with the calculated molecular mass of $45.8 \mathrm{kDa}$. Mass spectrometry with a sequence coverage of $74 \%$ confirmed that the purified protein was the expected product and that the C-terminus was intact (Fig. S5b). Circular dichroism (CD) spectroscopy revealed a high percentage of regular secondary structure elements, with $\alpha$-helices and $\beta$-sheets amounting to about $24 \%$ each (Fig. S5c). These values deviate from the values of $10 \%$-helix 
and $34 \% \beta$-sheet derived from the crystal structure of the protein (PDB 4YY8), which might suggest a higher percentage of $\alpha$-helices in solution as compared with the crystal. Interestingly, CD spectroscopy of the structurally similar protein Keap1 also suggested a flexible structure ${ }^{24}$ and a higher $\alpha$-helix content in solution than in the crystal structure ${ }^{34}$. We also probed the thermostability of the folded state of the protein by monitoring its secondary structure composition as a function of temperature with the aid of CD spectroscopy (Fig. S5d). Irrespective of the wavelength, a sharp transition was observed within a narrow temperature interval around $55^{\circ} \mathrm{C}$. Above this temperature, the $\mathrm{CD}$ signal decreased in magnitude and virtually disappeared even at those wavelengths where an unfolded polypeptide chain would manifest in a pronouncedly negative ellipticity (e.g., $192 \mathrm{~nm}$ and $198 \mathrm{~nm}$ ). This finding is in agreement with the observed protein precipitation at temperatures above $50^{\circ} \mathrm{C}$. Correct protein folding was corroborated independently by measuring the accessibility of cysteine residues. In accordance with the predicted accessibility of two out of seven cysteine residues (Fig. 4a), only two $(1.8 \pm 0.2)$ cysteine residues were found to be accessible to Ellman's reagent under native conditions, whereas all seven $(6.8 \pm 0.4)$ cysteine residues became accessible under denaturing conditions (Fig. S5d). Gel filtration chromatography revealed the presence of multiple oligomeric states (Fig. S5e). We detected either monomeric recombinant PfKelch13337-726 in $50 \mathrm{mM}$ sodium phosphate buffer without salt or the interconversion of monomeric, dimeric, and tetrameric protein in the same buffer containg $300 \mathrm{mM} \mathrm{NaCl}$, suggesting a dynamic equilibrium between these three species.

In summary, we generated a specific antibody and established a denaturing pull-down assay for PfKelch13, developed a protocol for the purification of soluble recombinant PfKelch13 ${ }^{337-726}$ from Sf21 cells, confirmed that two of the seven cysteine residues are surface exposed, and detected an unexpectedly flexible quaternary structure.

\section{Potential post-translational modification of PfKelch13}

Even though recombinant His-tagged full-length PfKelch13 produced in E. coli and Sf21 cells was insoluble, lysates allowed us to compare the electrophoretic mobility with PfKelch13 from 
P. falciparum by SDS-PAGE and western blot analysis. While unmodified PfKelch13 from strain 3D7 was detected at $95 \mathrm{kDa}$, His-tagged full-length PfKelch13 from strain $\mathrm{His}_{8}{ }^{-}$ PFKELCH13 $3^{\text {wt }}$ had an apparent molecular mass of approximately $97 \mathrm{kDa}$ (Fig. S6a). The mass difference between both proteins was in accordance with the modification of the $\mathrm{N}$-terminus of His ${ }_{8}$-PfKelch13 ${ }^{\text {wt }}$ due to the ribosomal skipping and His-tag, however, both proteins ran about 10 to $12 \mathrm{kDa}$ higher than expected. The mass shift was detected in blots that were probed with our anti-PfKelch13 antibody and in blots that were probed with a commercial antibody against the His-tag. Recombinant His-tagged PfKelch13 from E. coli or Sf21 cells was detected around $85 \mathrm{kDa}$ in accordance with the calculated molecular mass (Fig. S6a). These controls disproved an inherent abnormal mobility of PfKelch13 and confirmed the mass shift of PfKelch13 in $P$. falciparum. A second band with variable intensity around the expected molecular mass of 85 kDa was also detected in P. falciparum lysates that were probed with the anti-His antibody (Fig. 2b) or the anti PfKelch13 antibody (Fig. S6a). Although both PfKelch13 species were regulated in the knockdown experiments (Fig. 2b), the intensity of the signal at $85 \mathrm{kDa}$ and the ratio between the upper and lower band differed among experiments, suggesting a dynamic interconversion of the protein species during blood-stage development.

The modified gene architectures of $\mathrm{His}_{8}-$ PFKELCH13-gImS (Fig. 1a) and His ${ }^{-}$ PFKELCH13 ${ }^{\text {wt }}$ (Fig. S3a) refuted an alternative translation initiation site that is encoded at the 5' end of PFKELCH13. A putative translational read-through until the next stop codon would have increased the mass by just $1.8 \mathrm{kDa}$. Thus, a post-translational modification is the most plausible explanation for the mobility shift of PfKelch13 in P. falciparum. Since pull-down experiments worked well with our anti-PfKelch13 antibody (Fig. S4d), we excised the band corresponding to PfKelch13 and analyzed it by mass spectrometry (Fig. S6b). Although highest signal intensities corresponded to peptides of PfKelch13, we neither detected proteins nor post-translational modifications that could explain the altered electrophoretic mobility. In summary, we identified two different forms of PfKelch13 with distinct electrophoretic mobilities around 85 and $95 \mathrm{kDa}$ in $P$. falciparum, suggesting an unidentified post-translational modification. 


\section{Protein folding rather than redox properties correlates with artemisinin susceptibility}

Next, we produced single point mutants of $\mathrm{MH}_{8} \mathrm{SR}$-tagged PfKelch13 $3^{337-726}$ in Sf21 cells to analyze the altered protein properties. Mutations R539T, I543T and C580Y cause a decreased susceptilibity against artemisinin in the field and in cell culture ${ }^{7,9,15,31}$ and are all within the propeller domain (Fig. 5a). Furthermore, we systematically replaced the seven cysteine residues (Fig. 5b). Western blot analysis of the Sf21 lysates with the according recombinant proteins revealed that each of the field mutations had a strong destabilizing effect, resulting in completely insoluble recombinant PfKelch13 ${ }^{337-726}$ (Fig. 5c). This was also observed for the replacement C580F, whereas single replacements C447S, C469S, C532S, C542S, C580S, and C696S had no or only moderate effects on the protein solubility. An anomaly with intermediate solubility was observed for the replacement C473S. Protein purifications confirmed the different solubilities (Fig. 5d). In summary, not the loss of the disulfide bond between residues C532 and C580 but the C580Y or C580F replacement led to insoluble PfKelch13337-726. Impaired protein folding of recombinant PfKelch13337-726 correlates with the observed decreased artemisinin susceptibility of field mutants, suggesting that protein folding is the central common parameter for mutant selection.

\section{Discussion}

The relevance of PfKelch13 as an artemisinin susceptibility factor can be viewed from two different perspectives, one with a focus on the chemical properties and mode of action of artemisinin and one with a focus on the properties and structure-function relationships of PfKelch13. The endoperoxide group of artemisinin is a prerequisite for its antimalarial activity ${ }^{35}$. While artemisinin itself seems to be not a peroxidase substrate ${ }^{33}$, heme-dependent one electron reduction of the endoperoxide bond results in a reactive radical that was shown to alkylate heme in cell culture and mice $\mathrm{e}^{36,37}$ as well as proteins in vitro or in cell culture ${ }^{36,38,39}$. Furthermore, artemisinin-dependent inhibition of hemozoin formation was suggested to cause 
oxidative stress ${ }^{40}$. Thus, artemisinin might either directly or indirectly modify C580 or another cysteine residue in PfKelch13. A direct alkylation of PfKelch13 by artemisinin seems less likely considering the absent co-localization of PfKelch13, heme, and highly reactive artemisinin radicals. Modifications of specific cysteine residues of the vertebrate homologue Keap1 by hydrogen peroxide or alkylating agents is a key event in signal transduction that leads to the transcription of numerous genes and an increased biosynthesis of glutathione ${ }^{21}$. Rodent and human Keap1 have 25 and 27 cysteine residues, respectively. Two of these residues in the intervening region were shown to act as a sensor and to react with electrophiles ${ }^{22,41-43}$. Another regulatory cysteine that is activated by a different set of electrophiles was found in the BTB domain, suggesting a highly complex integration of diverse signals $22,24,42,43$. The homology between Keap1 and PfKelch13 leads to the hypothesis that PfKelch13 also acts as a redox sensor. Mutations in PFKELCH13 were indeed shown to correlate with an increased abundance of $y$-glutamylcysteine and glutathione ${ }^{44}$. However, PfKelch13 lacks the intervening region of Keap1, all its seven cysteine residues are located in the $\beta$-propeller domain, and serine replacements for C469, C532 or C580 of PfKelch13 had no effect on the artemisinin susceptibility in cell culture. Thus, a potential redox regulation of these residues is irrelevant for the altered artemisinin susceptibility. If PfKelch13 undergoes an essential redox regulation and/or an artemisinin-dependent redox regulation, it has to depend on the thiol group of residue C473, which was refractory to replacement in $P$. falciparum blood stages and which also affected the folding properties of recombinant PfKelch13.

We provide evidence that PfKelch13 variants in the field have an altered protein stability (Fig. 6). We established a protocol for the production of recombinant PfKelch13 and showed that each of the common mutations R539T, I543T or C580Y destabilizes the protein and decreases its solubility in accordance with molecular dynamics simulations ${ }^{10}$. While mutations C580S or C532S had no effect on protein folding, mutations R539T, I543T, C580Y or C580F all resulted in misfolded insoluble recombinant proteins. Thus, protein stability and abundance link the previously detected decreased or normal artemisinin susceptibility following protein mislocalization of wild-type PfKelch13 or overexpression of PFKELCH13 ${ }^{C 580 Y}$, respectively ${ }^{15-}$ 
17. A correlation between protein abundance and artemisinin susceptibility was previously shown by quantitative mass spectrometry ${ }^{17,44}$. We now confirmed the link between protein abundance and artemisinin susceptibility using a titratable knockdown system (Fig. 6). The knockdown resulted in arrested ring-stage parasites during the second intraerythrocytic cycle. This result is in accordance with previous mislocalization studies showing that PfKelch13 is not essential for schizont development ${ }^{16}$. The developmental arrest was shown to correlate with a decreased PfKelch13-dependent endocytotic uptake and proteolytic degradation of hemoglobin in early ring-stage parasite ${ }^{16,17,44}$, which is thought to prevent the hemedependent activation of artemisinin and to give the parasite time to survive the peak concentration of the rather labile drug ${ }^{6,40,45}$. Our knockdown parasites reached ring-stage survival percentages slightly above $40 \%$. This value might indicate a physiological threshold because knockdown parasites died at higher GlcN concentrations and similar maximum survival percentages were reported for RSAs with dihydroartemisinin and mutants R539T and I543T in other genetic backgrounds ${ }^{9}$. The degree of misfolding of common $\beta$-propeller domain mutants in $P$. falciparum remains to be analyzed. However, misfolding of the $\beta$-propeller domain or decreasing the amount of PfKelch to less than $10 \%$ was still compatible with the essential function of PfKelch13, which was shown to require the $\mathrm{N}$-terminal apicomplexanspecific region ${ }^{16}$. Lower PfKelch13 abundances presumably limit the endocytotic uptake of nutrients to such a degree that the parasite cannot develop any further.

Our comparative western blot analyses with recombinant and endogenously tagged as well as untagged PfKelch13 revealed two different forms of PfKelch13 with distinct electrophoretic mobilities around 85 and $95 \mathrm{kDa}$. It is unlikely that the double band and mass shift were caused by limited proteolysis as the C-terminus of PfKelch13 is buried according to PDB entry 4YY8 and the N-terminal His-tag was detected for both recombinant and endogenously tagged PfKelch13. Since we can also exclude an alternative translation initiation site or a translational read-through as potential causes for the mass shift, we suggest that a major fraction of PfKelch13 undergoes a post-translational modification. The potential modification remains to be identified. Attachment of a protein, such as SUMO or ubiquitin, appears to be the most likely 
modification, not only because of the mass shift but also because PfKelch13 lacks common peptide motifs for lipid modifications (and multiple phosphorylations or acetylations would rather increase the electrophoretic mobility due to additional negative charges or the loss of positive charges, respectively). It also remains to be determined whether a post-translational modification regulates (i) the alternative oligomeric states that were observed for recombinant PfKelch13 in vitro, (ii) protein-protein interactions, and/or (iii) the membrane recruitment of PfKelch13 to different subcellular compartments, including the doughnut-shaped structures at cytostomes $^{16-18}$ (Fig. 6).

In conclusion, we showed that down-regulation of PfKelch13 results in ring-stage survival rates of up to $40 \%$ and that common field mutations have a destabilizing effect on the folding properties of PfKelch13. We also showed that PfKelch13 exists in at least two different forms and established a protocol for the production of recombinant PfKelch13 ${ }^{337-726}$. The recombinant protein adopts alternative oligomeric states and two of its seven cysteine residues react with DTNB. While the thiol group of C473 of PfKelch13 appears to be essential for blood-stage development, the redox state of C580 and C532 is irrelevant for the PfKelch13-dependent artemisinin susceptibility.

\section{Online methods}

Cloning of constructs. Primers were purchased from Metabion and are listed in Supplementary Tables S2 - S4. SLI constructs were generated using vector pSLI-N-GFP2xFKBP-loxP(K13) ${ }^{15}$ (addgene plasmid \#85792). Insert GFP-2xFKBP-K13 was excised with Nhel and Xhol. Recodonized PFKELCH13 was amplified by PCR using primers P65 and P69 and vector pSLI-N-GFP-2xFKBP-loxP(K13) as a template. The PCR product was digested with Sall and Xhol. Primers P60 and P61 that encode the Hiss-tag were annealed, yielding cohesive ends for Nhel and Sall. The annealed primers were ligated with the digested vector and PCR product, yielding pSLI-His ${ }_{8}-P F K E L C H 13$. To introduce mutations in recodonized PFKELCH13, 
the gene was first subcloned into plasmid pET45b using restriction sites Avrll and Xhol, followed by site-directed mutagenesis with primers P71 - P76 or P84 - P87, and recloning into pSLI-N-GFP-2xFKBP-loxP(K13) using primers P65 and P69. Primers P88 and P89 were used to amplify the gImS and $M 9$ sequences from plasmids pCR 2.1 TOPO- $m$ Cherry-gImS and pCR 2.1 TOPO-mCherry-M9, respectively ${ }^{46}$. The loxP site from pSLI-His 8 -PFKELCH13 was subsequently excised and replaced with the glmS or M9 sequence using Stul and Xhol, yielding pSLI-His ${ }_{8}$-PFKELCH13-gImS and pSLI-His ${ }_{8}$ PFKELCH13-M9.

Plasmid pET45b-PFKELCH13 with synthetic PFKELCH13 that was codon-optimized for the expression in E. coli (Fig. S7) was purchased from GenScript and was used as a template to PCR-amplify truncated PFKELCH13 $337-726$ with primers P1 and P2. The PCR product was cloned into the Kpnl and Avrll restriction sites of pET45b, resulting in construct pET45bPFKELCH13 ${ }^{337-726}$. Plasmids pET45b-PFKELCH13 and pET45b-PFKELCH13 $3^{337-726}$ served as templates for site-directed mutagenesis with primers P6 - P23 to generate serine mutants for each of the seven cysteine residues as well as mutants C580Y and C580F. Synthetic PFKELCH13 and PFKELCH13 $337-726$ from plasmid pET45b were also PCR-amplified and subcloned into the BamHI and HindIII restriction sites of pQE30 (using primers P3 - P5), the BamHI and Notl restriction sites of pGEX-4T-1 (using primers P3, P4 and P30), the Ndel and Notl restriction sites of pMAL-c5X (using primers P30, P32 and P33), the EcoRI and Notl restriction sites of pBLHIS-SX (using primers P30, P37 and P39), and the Xbal and Notl restriction sites of pLEXSY-IE-blecherry4 (using primers P30, P36 and P38).

Constructs for expression in Sf21 cells were based on plasmid pCoofy41 (addgene plasmid \#55184) $)^{47}$, which was digested with BamHI and HindIII. PFKELCH13 $3^{337-726}$ was amplified by PCR from wild-type and mutant pET45b-PFKELCH13 using primers P26 and P4. The PCR products were digested with Xbal and HindIII. Primers P50 and P51 that encode the His 8 -tag were annealed, yielding cohesive ends for BamHI and $\mathrm{Xbal}$. The annealed primers were ligated with the digested vector and PCR product, yielding wild-type or cysteine mutantencoding pCoofy-His 8 -PFKELCH13 $3^{337-726}$. Two additional mutant versions (R539T and I543T) were generated by site-directed mutagenesis with primers P95 - P98 using pCoofy-His ${ }^{-}$ 
PFKELCH13 $3^{337-726}$ as a template. Construct pCoofy-His ${ }_{8}$ PFKELCH13 encoding full-length PfKelch13 was generated analogously with primers P52 and P4. All constructs were confirmed by Sanger sequencing (SEQ-IT GmbH \& Co. KG).

P. falciparum culture, transfection and selection. $P$. falciparum strains were cultured according to Trager and Jensen ${ }^{48}$ with slight modifications ${ }^{49}$ at $37^{\circ} \mathrm{C}, 5 \% \mathrm{O}_{2}, 5 \% \mathrm{CO}_{2}$, and $90 \%$ humidity in fresh human $A^{+}$erythrocytes at a hematocrit of $3.6 \%$ in complete RPMI-1640 medium containing $0.45 \%$ AlbuMAX II, $0.2 \mathrm{mM}$ hypoxanthine, and $5 \mu \mathrm{g} / \mathrm{mL}$ gentamicin. Standard $14 \mathrm{~mL}$ cultures were maintained in vented petri dishes.

Transfections were performed with pre-loaded uninfected erythrocytes that were subsequently infected with $P$. falciparum ${ }^{50,51}$. Parasites were synchronized with $5 \%$ sorbitol ${ }^{52}$ one day before transfection. To sterilize the plasmid DNA, $100 \mu \mathrm{g}$ of a DNA from a midipreparation was acidified with 0.1 volumes of buffer containing $3 \mathrm{M}$ sodium acetate/acetic acid, $\mathrm{pH} 4.7$ and precipitated with 2.5 volumes of absolute ethanol at $-20^{\circ} \mathrm{C}$ over night. After centrifugation at $20000 \times g$ for $30 \mathrm{~min}$ at $4^{\circ} \mathrm{C}$, the DNA was washed with $500 \mu \mathrm{L}$ of ice-cold $70 \%$ ethanol. The supernatant was discarded under sterile conditions and the DNA was dried for $10 \mathrm{~min}$ before the transfection. Fresh erythrocytes were washed twice with 3.5 volumes of cytomix containing $120 \mathrm{mM} \mathrm{KCl}, 5 \mathrm{mM} \mathrm{MgCl}_{2}, 0.15 \mathrm{mM} \mathrm{CaCl}_{2}, 2 \mathrm{mM} \mathrm{EGTA}, 10 \mathrm{mM} \mathrm{K}_{2} \mathrm{HPO}_{4}$, $10 \mathrm{mM} \mathrm{KH} \mathrm{PO}_{4}, 25 \mathrm{mM}$ HEPES/KOH, $\mathrm{pH} 7.6$ at room temperature. An aliquot of $400 \mu \mathrm{L}$ washed erythrocytes was mixed with $100 \mu \mathrm{g}$ of sterile DNA in $400 \mu \mathrm{L}$ cytomix, transferred to two electroporation cuvettes, incubated on ice for $5 \mathrm{~min}$, and electroporated using program $\mathrm{U}$ 033 of the Nucleofector $2 b$ (Lonza). Following electroporation, both cuvettes were immediately incubated for 5 min on ice. Pre-loaded erythrocytes were combined after rinsing each cuvette with $4 \mathrm{~mL}$ complete RPMI-1640 medium, centrifuged at $300 \times \mathrm{g}$ for $5 \mathrm{~min}$, and resuspended together with $100 \mu \mathrm{L}$ infected erythrocytes with $1 \%$ parasitemia in complete RPMI-1640 medium. Cultures were washed the next day and supplemented with $5 \mathrm{nM}$ of the antifolate WR99210 in a new petri dish. Drug and medium were changed daily during the first week. Afterwards, WR99210 and medium were renewed every other day, and $50 \mu \mathrm{L}$ of fresh 
erythrocytes were added once a week until parasites were detected by light microscopy in Giemsa-stained blood smears 3-5 weeks post-transfection.

SLI was performed with successfully transfected and selected parasites containing pSLI plasmids ${ }^{15}$. Cultures were grown to a parasitemia of $5 \%$ and supplemented with $0.9 \mu \mathrm{M}$ DSM1. Drug and medium were renewed daily. Usually, parasites disappeared after 4 days and reemerged after around 2 weeks. Successful integration was confirmed by genotyping PCRs with extracted genomic DNA ${ }^{53}$ and western blot analysis against the His ${ }_{8}$-tag following parasite isolation by saponin treatment ${ }^{54}$.

Growth curve analyses, $I_{50}$ measurements and RSAs. A $1.16 \mathrm{~mL}$ stock solution of 400 mM GICN was prepared freshly by neutralizing $100 \mathrm{mg} \mathrm{GlcN}{ }^{\star} \mathrm{HCl}$ (Sigma) with $232 \mu \mathrm{L} 2 \mathrm{M}$ $\mathrm{NaOH}$ and adding $928 \mu \mathrm{L}$ RPMI-1640 medium. Asynchronous cultures of strains His $8^{-}$ PFKELCH-glmS and His ${ }_{8}$-PFKELCH-M9 were set to a parasitemia of $0.1 \%$, split into four and treated with either $0,0.3,1.0,2.0,5.0$ or $10 \mathrm{mM} \mathrm{GlcN}$ for five days. The medium with the corresponding GlcN concentration was replaced once a day. Giemsa-stained blood smears were analyzed by light microscopy. At least 2000 erythrocytes were counted to determine the parasitemia for each day. The data from at least three independent growth experiments was analyzed in SigmaPlot13.

$I_{50}$ measurements were performed in a SYBR green I plate-reader assay as described previously ${ }^{32,55}$ with slight modifications. Parasite cultures were synchronized twice with sorbitol. Stock solutions of $0.49 \mu \mathrm{M}$ artesunate, $39 \mathrm{mM} \mathrm{tBuOOH}, 49 \mathrm{mM}$ diamide, $122 \mathrm{mM}$ DTT, and $97 \mu \mathrm{M}$ DSM1 were freshly prepared in albumax-free RPMI-1640 medium containing $0.2 \mathrm{mM}$ hypoxanthine and $5 \mu \mathrm{g} / \mathrm{mL}$ gentamicin, and were subsequently filter-sterilized. Early ring-stage parasites in $100 \mu \mathrm{L}$ complete medium at a parasitemia of $0.3 \%$ and a hematocrit of $1.5 \%$ were treated in 96-well plates with $0.01-81 \mathrm{nM}$ artesunate, $1.0 \mu \mathrm{M}-6.5 \mathrm{mM} \mathrm{tBuOOH}, 1.2 \mu \mathrm{M}-$ 8.1 mM diamide, $3.0 \mu \mathrm{M}-20 \mathrm{mM}$ DTT, or $2.5 \mathrm{nM}-16 \mu \mathrm{M}$ DSM1, incubated for $140 \mathrm{~h}$, and then stored at $-80^{\circ} \mathrm{C}$. Uninfected erythrocytes as well as untreated parasites served as controls. Cells were thawed and lysed in $100 \mu \mathrm{L}$ lysis buffer $(20 \mathrm{mM}$ Tris- $\mathrm{HCl}, \mathrm{pH} 7.5,5 \mathrm{mM}$ 
EDTA, $0.08 \%(\mathrm{v} / \mathrm{v})$ Triton $\mathrm{X}-100,0.008 \%(\mathrm{w} / \mathrm{v})$ saponin) that was supplemented with 0.12 $\mu \mathrm{L} / \mathrm{mL} 10000 \times$ SYBR green I solution, and the fluorescence was analyzed using a ClarioStar plate reader (BMG Labtech) with an excitation at $485 \mathrm{~nm}$ and an emission at $535 \mathrm{~nm}$. The gain and optimal measuring height were internally adjusted to the highest expected fluorescence intensities for the parasite cultures without drug. The fluorescence intensities of the uninfected erythrocytes with the corresponding drug concentrations were substracted from the intensities of the parasite cultures. The resulting fluorescence intensities were normalized with regard to the maximum intensities from untreated controls, plotted against the corresponding drug concentrations, and fitted to a sigmoidal dose-response curve using a four-parameter Hill function in SigmaPlot13.

RSAs were performed as described previously ${ }^{4,33}$ with slight modifications. Early ring-stage cultures were highly synchronized by three consecutive sorbitol treatments with a time difference of $45 \mathrm{~h}$. The synchronized culture was split in two and set to a parasitemia of $1 \%$ and a hematocrit of $2 \%$. One $14 \mathrm{~mL}$ culture was treated with $700 \mathrm{nM}$ artesunate. The other culture was treated with the same DMSO concentration and served as a control to calculate the survival percentage. After $6 \mathrm{~h}$, the cultures were washed four times with $5 \mathrm{~mL}$ complete RPMI-1640 medium, resuspended in $14 \mathrm{~mL}$ complete RPMI-1640 medium, and cultured for another $66 \mathrm{~h}$. The parasitemia was determined from Giemsa-stained blood smears.

The formation of hemozoin was analyzed for highly synchronized strains His - PFKELCHgImS and Hiss-PFKELCH-M9. One cycle before the assay, parasites were pre-synchronized with sorbitol and incubated with 50 units of heparin to inhibit invasion. When the majority of parasites presented as segmenters, heparin was washed off and invasion was allowed for $2 \mathrm{~h}$ under constant agitation at $80 \mathrm{rpm}$. All remaining late-stage parasites were removed by sorbitol treatment and cultures were treated with $1 \mathrm{mM}$ GlcN. Medium with GlcN was replaced once per day and thin blood smears were taken at 18-28 $\mathrm{h}$ hpi every 2 hours for the first cycle. After the $28 \mathrm{hpi}$ timepoint of the first cycle, cultures were again treated with 50 units of heparin. When the majority of parasites presented as segmenters, invasion was allowed for $1 \mathrm{~h}$ at 80 rpm. All remaining late-stage parasites were removed by sorbitol treatment. For the second 
cycle, thin blood smears were taken at 16-32 hpi every 2 hours. Parasites were examined for hemozoin by light microscopy of thin blood smears that were stained with Hemacolor rapid staining of blood smear solution (Sigma) using a BZ-X800 microscope (Keyence).

Recombinant PfKelch13 and antibody generation. To produce His 8 -tagged PfKelch13 and PfKelch13337-726 in E. coli, the full-length and truncated constructs in pET45b, pQE30, pGEX4T-1 and pMAL-c5X were tested using the strains and conditions listed in supplementary Table 1. Recombinant PfKelch13337-726 for the generation of antibodies was produced in E. coli SHuffle T7 Express cells (NEB) containing pET45b-PFKELCH13 $3^{337-726}$. Cells were grown in an Innova $44 \mathrm{R}$ shaker at $170 \mathrm{rpm}$ at $30^{\circ} \mathrm{C}$ and induced with $0.5 \mathrm{mM}$ isopropyl $\beta-\mathrm{D}-1$ thiogalactopyranoside (IPTG) at an opitical density (OD) of 0.5 . After $4 \mathrm{~h}$, cultures were incubated for $10 \mathrm{~min}$ in an ice-water bath and centrifuged in a Beckman JS-4.2 rotor at 4000 $\times g$ for $15 \mathrm{~min}$ at $4^{\circ} \mathrm{C}$. The cell pellet from $1 \mathrm{~L}$ culture was resuspended in $10 \mathrm{~mL}$ ice-cold purification buffer ( $\left.300 \mathrm{mM} \mathrm{NaCl}, 50 \mathrm{mM} \mathrm{Na}_{x} \mathrm{H}_{\mathrm{y}} \mathrm{PO}_{4}, \mathrm{pH} 8.0\right)$ and stored at $-20^{\circ} \mathrm{C}$. The thawed suspension was supplemented with $10 \mathrm{mg}$ lysozyme and a spatula tip of DNase, stirred on ice for $1 \mathrm{~h}$, sonicated on ice and centrifuged at $10000 \times g$ for $30 \mathrm{~min}$ at $4^{\circ} \mathrm{C}$. The pellet containing the inclusion bodies was washed twice at room temperature with $10 \mathrm{~mL}$ washing buffer (300 $\mathrm{mM} \mathrm{NaCl}, 2 \%(\mathrm{w} / \mathrm{v})$ Triton $\mathrm{X}-100,2 \mathrm{M}$ urea, $50 \mathrm{mM}$ Tris- $\mathrm{HCl}, \mathrm{pH} 8.0)$ followed by sonication and solubilization in $10 \mathrm{~mL}$ solubilization buffer $(300 \mathrm{mM} \mathrm{NaCl}, 2 \%$ (w/v) SDS, $8 \mathrm{M}$ urea, 50 $\mathrm{mM}$ Tris- $\mathrm{HCl}, \mathrm{pH} 8.0)$. Excess SDS was precipitated at $4^{\circ} \mathrm{C}$ overnight followed by centrifugation at $10000 \times g$ for $30 \mathrm{~min}$ at $4^{\circ} \mathrm{C}$. The supernatant was loaded on a $500 \mu \mathrm{L} \mathrm{Ni-NTA}$ agarose column (Qiagen), washed with $10 \mathrm{~mL}$ solubilization buffer without SDS and eluted with $1.5 \mathrm{~mL}$ of the same buffer containing $200 \mathrm{mM}$ imidazole, $\mathrm{pH}$ 8.0. Eluted PfKelch13337-726 was washed and concentrated to $60 \mu \mathrm{M}$ in solubilization buffer without SDS using a Amicon Ultra-15, PLGC Ultracel-PL Membran, $10 \mathrm{kDa}$ unit (Merck) and heated at $95^{\circ} \mathrm{C}$ for $10 \mathrm{~min}$ in the presence of $40 \mathrm{mM}$ DTT to reduce all cysteines. The solubilized denatured protein from $E$. coli was used for commercial immunization of rabbits (Pineda Antikörper Service, Berlin) and was also 
coupled to $\mathrm{CNBr}$-activated sepharose ${ }^{56}$ for the purification of PfKelch13 antibodies from rabbit serum by affinity-chromatography.

Recombinant Hiss-tagged PfKelch13 and PfKelch13 ${ }^{337-726}$ were produced in Sf21 cells that were routinely cultured in Sf900 ${ }^{\mathrm{TM}}$ III SFM medium (Thermo) in an Innova 44R shaker at 120 rpm at $27^{\circ} \mathrm{C}$. The PFKELCH13-encoding pCoofy constructs were used for transposition into E. coli DH10EMBacY cells (Geneva Biotech) and bacmid DNA was isolated from $3 \mathrm{~mL}$ overnight cultures. For the transfection, a solution containing $10 \mu \mathrm{g}$ of bacmid DNA and $50 \mu \mathrm{L}$ of medium were mixed with a second solution comprising $5 \mu \mathrm{L}$ of X-tremeGENE HP DNA transfection reagent (Merck) and $50 \mu \mathrm{L}$ medium. The transfection mixture was incubated for $10 \mathrm{~min}$ at room temperature and then added dropwise to $0.6 \times 10^{6}$ adherent Sf21 cells in $2 \mathrm{~mL}$ medium in a 6-well Corning plate. After $5 \mathrm{~h}$ of incubation at $27^{\circ} \mathrm{C}, 1 \mathrm{~mL}$ of medium was added to each well and the plate was incubated for 3 days at $27^{\circ} \mathrm{C}$. The cell culture medium containing the $V_{0}$ baculovirus stock was harvested and $2 \mathrm{~mL}$ of $V_{0}$ was used to infect $25 \mathrm{~mL} \mathrm{Sf} 21$ cells at a density of $0.6 \times 10^{6}$ cells $/ \mathrm{mL}$ in a $125 \mathrm{~mL}$ Erlenmeyer flask to generate the $\mathrm{V}_{1}$ baculovirus stock. Once the cells stopped proliferating, they were kept in culture for an additional $24 \mathrm{~h}$ and then the baculovirus $\left(\mathrm{V}_{1}\right.$ stock) was harvested by centrifugation $(10 \mathrm{~min}, 550 \times \mathrm{g})$. For recombinant protein production, $5 \mathrm{~mL}$ of $\mathrm{V}_{1}$ was added to $1 \mathrm{~L}$ of $\mathrm{Sf} 21$ culture at a density of $10^{6} \mathrm{cells} / \mathrm{mL}$. After $72 \mathrm{~h}$, cells were harvested $(10 \mathrm{~min}, 650 \times \mathrm{g})$ and stored at $-20^{\circ} \mathrm{C}$. The cell pellet was resuspended in $30 \mathrm{~mL}$ ice-cold lysis buffer $(20 \mathrm{mM}$ imidazole, $300 \mathrm{mM} \mathrm{NaCl}, 10 \mathrm{mM}$ $\mathrm{MgCl}_{2}, 1$ spatula tip DNase, 1 tablet cOmplete EDTA-free (Roche), 50 mM Na $\mathrm{H}_{\mathrm{y}} \mathrm{PO}_{4}, \mathrm{pH}$ 8.0) and divided into four samples. Cells were disrupted by three freeze-thaw cycles using liquid nitrogen and centrifuged at $10000 \times g$ for $30 \mathrm{~min}$ at $4^{\circ} \mathrm{C}$. The combined supernatants were loaded on a $0.8 \mathrm{~mL}$ Ni-NTA agarose column that was equilibrated with $10 \mathrm{~mL}$ buffer containing $20 \mathrm{mM}$ imidazole, $300 \mathrm{mM} \mathrm{NaCl}, 50 \mathrm{mM} \mathrm{Na}_{x} \mathrm{H}_{\mathrm{y}} \mathrm{PO}_{4}, \mathrm{pH}$ 8.0. The column was washed with 10 $\mathrm{mL}$ ice-cold washing buffer (50 mM imidazole, $300 \mathrm{mM} \mathrm{NaCl}, 50 \mathrm{mM} \mathrm{Na}_{x} \mathrm{H}_{\mathrm{y}} \mathrm{PO}_{4}, \mathrm{pH}$ 8.0) and the protein was eluted with buffer containing $250 \mathrm{mM}$ imidazole, $300 \mathrm{mM} \mathrm{NaCl}, 50 \mathrm{mM}$ $\mathrm{Na}_{x} \mathrm{H}_{4} \mathrm{PO}_{4}, \mathrm{pH} 8.0$. 
Gel filtration chromatography, Ellman's assay and CD spectroscopy. The oligomerization state of recombinant PfKelch13 $3^{337-726}$ freshly purified from Sf21 cells was analyzed at $10^{\circ} \mathrm{C}$ on a Superdex 200 Increase 10/300 GL column connected to an Äkta explorer system using 50 $\mathrm{mM} \mathrm{Na} \mathrm{H}_{4} \mathrm{PO}_{4}, \mathrm{pH} 8.0$ with or without $300 \mathrm{mM} \mathrm{NaCl}$ as a running buffer.

The number of accessible cysteines in PfKelch13 ${ }^{337-726}$ was determined with Ellman's reagent 5,5'-dithiobis-(2-nitrobenzoic acid) $(\mathrm{DTNB})^{57}$. Freshly purified recombinant PfKelch13 ${ }^{337-726}$ was reduced with $5 \mathrm{mM}$ DTT for $30 \mathrm{~min}$ on ice. Imidazole and excess DTT were removed using a PD-10 desalting column and phosphate buffer (300 mM NaCl, $50 \mathrm{mM}$ $\mathrm{Na}_{x} \mathrm{H}_{\mathrm{y}} \mathrm{PO}_{4}, \mathrm{pH}$ 8.0). The protein was diluted with phosphate buffer to a concentration of $10 \mu \mathrm{M}$ and $30 \mu \mathrm{M}$ using the calculated molar extinction coefficient of $\varepsilon_{280 \mathrm{~nm}}=59.82 \mathrm{mM}^{-1} \mathrm{~cm}^{-1}$. Samples were mixed with equal volumes of $1.2 \mathrm{mM}$ DTNB in either phosphate buffer or denaturing buffer (2\% (w/v) SDS, $\left.300 \mathrm{mM} \mathrm{NaCl}, 50 \mathrm{mM} \mathrm{Na}_{x} \mathrm{H}_{\mathrm{y}} \mathrm{PO}_{4}, \mathrm{pH} 8.0\right)$. After 5 min incubation at room temperature, the absorbance was measured at $412 \mathrm{~nm}$ using a JASCO V-650 UV/Vis spectrophotometer. Calibration curves with $0-40 \mu \mathrm{M}$ DTT were generated in parallel for both buffers and were used to calculate the concentration of accessible thiols under native and denaturing conditions from three independent protein purification experiments.

For CD spectroscopy, freshly purified recombinant PfKelch13 $3^{337-726}$ was concentrated and the buffer exchanged with CD buffer (100 mM NaF, 50 mM Nax $\mathrm{H}_{y} \mathrm{PO}_{4}, \mathrm{pH} 7.4$ ) in an Amicon Ultra-15, PLGC Ultracel-PL Membran, $10 \mathrm{kDa}$ unit. CD spectra were recorded at $20^{\circ} \mathrm{C}$ using a thermostatted Chirascan CD spectrometer (Applied Photophysics). The proportions of secondary structure elements were estimated from the spectra using the K2D2 software ${ }^{58}$. The contents of secondary structure elements in the crystal structure of PfKelch13 $337-726$ (PDB entry 4YY8) were calculated using the webserver 2StrucCompare.

Immunoprecipitation of PfKelch13. For immunoprecipitation of PfKelch13 from $P$. falciparum, four $14 \mathrm{ml}$ blood-stage cultures with a parasitemia of $\sim 10 \%$ trophozoites were combined and harvested by centrifugation at $300 \times g$ for $5 \mathrm{~min}$. Following saponin treatment ${ }^{54}$, parasites were resuspended in $250 \mu \mathrm{L}$ lysis buffer $(150 \mathrm{mM} \mathrm{NaCl}, 2 \%(\mathrm{w} / \mathrm{v}) \mathrm{SDS}, 1 \%(\mathrm{v} / \mathrm{v})$ Triton $\mathrm{X}-100,10 \mathrm{mM}$ Tris- $\mathrm{HCl}, \mathrm{pH}$ 7.5) containing cOmplete EDTA-free protease inhibitor 
(Roche) and frozen in liquid nitrogen. After thawing, the lysate was cleared by centrifugation at $16000 \times g$ for $10 \mathrm{~min}$ at room temperature. The supernatant was diluted 1:20 with buffer containing $150 \mathrm{mM} \mathrm{NaCl}, 10 \mathrm{mM}$ Tris- $\mathrm{HCl}, \mathrm{pH} 7.5$ and incubated at $4^{\circ} \mathrm{C}$ overnight rotating end over end with PfKelch13 antibody that was coupled to $100 \mu \mathrm{L} \mathrm{CNBr-activated} \mathrm{sepharose.} \mathrm{The}$ sepharose was centrifuged at $500 \times g$ for $5 \mathrm{~min}$ at $4^{\circ} \mathrm{C}$ and washed three times with $1 \mathrm{~mL} 150$ $\mathrm{mM} \mathrm{NaCl}, 10 \mathrm{mM}$ Tris- $\mathrm{HCl}, \mathrm{pH}$ 7.5. Bound proteins were eluted by adding $100 \mu \mathrm{L}$ of $2 x$ Laemmli buffer and heating at $95^{\circ} \mathrm{C}$ for $5 \mathrm{~min}$. Samples were subjected to SDS-PAGE and western blot analysis.

\section{Data availability}

All relevant data are included in the manuscript or its supplementary information and are available from the authors upon request.

\section{Acknowledgments}

This work was funded by the Deutsche Forschungsgemeinschaft (DFG, German Research Foundation) through the priority program SPP 1710 (grant DE 1431/8-2 to M.D.) and the SFB 1129 (project number 25240245660 to M.G.) as well as the Baden-Württemberg Foundation (ref: 1.16101 .17 to M.G.). We thank Frederik Sommer and Michael Schroda for the mass spectrometry analysis of recombinant PfKelch13 ${ }^{337-726}$ from Sf21 cells, Markus Räschle for the mass spectrometry analysis of purified His,-Kelch13 from $P$. falciparum, Simone Eggert and

Stefan Kins for their support with PfKelch13 expression trials in P. pastoris, and Tobias Spielmann for SLI plasmids and helpful discussions. 


\section{Author Contributions}

M.D. and R.S. conceived the study design. R.S. conducted and M.D. supervised all experiments unless otherwise indicated. The hemozoin analysis was conceived and supervised by M.G. and performed by S.KI., S.Ke. supervised the CD spectroscopy, and K.R. supervised the establishment of the production of recombinant PfKelch13 in Sf21 cells by R.S. and J.F.. R.S. and E.B. performed the growth curve analyses, protein purifications and DTNB assays. S.P. assisted with the cell culture, genotyping and western blot analysis. M.D. wrote the manuscript. All authors discussed the results and gave approval to the final version of the manuscript.

\section{Competing financial interests}

The authors declare no competing financial interests.

\section{References}

1. Organization, W.H. World Malaria Report 2015. http://www.who.int/malaria/publications/world-malaria-report-2015/report/en/ (2016).

2. Dondorp, A.M. et al. Artemisinin resistance in Plasmodium falciparum malaria. $N$ Engl J Med 361, 455-67 (2009).

3. Saralamba, S. et al. Intrahost modeling of artemisinin resistance in Plasmodium falciparum. Proc Natl Acad Sci U S A 108, 397-402 (2011).

4. Witkowski, B. et al. Novel phenotypic assays for the detection of artemisinin-resistant Plasmodium falciparum malaria in Cambodia: in-vitro and ex-vivo drug-response studies. Lancet Infect Dis 13, 1043-9 (2013).

5. Witkowski, B. et al. Reduced artemisinin susceptibility of Plasmodium falciparum ring stages in western Cambodia. Antimicrob Agents Chemother 57, 914-23 (2013).

6. Klonis, N. et al. Altered temporal response of malaria parasites determines differential sensitivity to artemisinin. Proc Natl Acad Sci U S A 110, 5157-62 (2013).

7. Ashley, E.A. et al. Spread of artemisinin resistance in Plasmodium falciparum malaria. N Engl J Med 371, 411-23 (2014).

8. Ariey, F. et al. A molecular marker of artemisinin-resistant Plasmodium falciparum malaria. Nature 505, 50-5 (2014). 
9. Straimer, J. et al. Drug resistance. K13-propeller mutations confer artemisinin resistance in Plasmodium falciparum clinical isolates. Science 347, 428-31 (2015).

10. Coppee, R., Jeffares, D.C., Miteva, M.A., Sabbagh, A. \& Clain, J. Comparative structural and evolutionary analyses predict functional sites in the artemisinin resistance malaria protein K13. Sci Rep 9, 10675 (2019).

11. Mathieu, L.C. et al. Local emergence in Amazonia of Plasmodium falciparum k13 C580Y mutants associated with in vitro artemisinin resistance. Elife 9(2020).

12. Miotto, O. et al. Emergence of artemisinin-resistant Plasmodium falciparum with kelch13 C580Y mutations on the island of New Guinea. PLoS Pathog 16, e1009133 (2020).

13. Uwimana, A. et al. Emergence and clonal expansion of in vitro artemisinin-resistant Plasmodium falciparum kelch13 R561H mutant parasites in Rwanda. Nat Med 26, 1602-1608 (2020).

14. Enserink, M. Malaria's drug miracle in danger. Science 328, 844-6 (2010).

15. Birnbaum, J. et al. A genetic system to study Plasmodium falciparum protein function. Nat Methods 14, 450-456 (2017).

16. Birnbaum, J. et al. A Kelch13-defined endocytosis pathway mediates artemisinin resistance in malaria parasites. Science 367, 51-59 (2020).

17. Yang, T. et al. Decreased K13 Abundance Reduces Hemoglobin Catabolism and Proteotoxic Stress, Underpinning Artemisinin Resistance. Cell Rep 29, 2917-2928 e5 (2019).

18. Gnadig, N.F. et al. Insights into the intracellular localization, protein associations and artemisinin resistance properties of Plasmodium falciparum K13. PLoS Pathog 16, e1008482 (2020).

19. Siddiqui, F.A. et al. Role of Plasmodium falciparum Kelch 13 Protein Mutations in P. falciparum Populations from Northeastern Myanmar in Mediating Artemisinin Resistance. mBio 11(2020).

20. Itoh, K. et al. Keap1 represses nuclear activation of antioxidant responsive elements by Nrf2 through binding to the amino-terminal Neh2 domain. Genes Dev 13, 76-86 (1999).

21. Brigelius-Flohe, R. \& Flohe, L. Basic principles and emerging concepts in the redox control of transcription factors. Antioxid Redox Signal 15, 2335-81 (2011).

22. Zhang, D.D. \& Hannink, M. Distinct cysteine residues in Keap1 are required for Keap1dependent ubiquitination of Nrf2 and for stabilization of Nrf2 by chemopreventive agents and oxidative stress. Mol Cell Biol 23, 8137-51 (2003).

23. Kobayashi, A. et al. Oxidative and electrophilic stresses activate Nrf2 through inhibition of ubiquitination activity of Keap1. Mol Cell Biol 26, 221-9 (2006).

24. Rachakonda, G. et al. Covalent modification at Cys151 dissociates the electrophile sensor Keap1 from the ubiquitin ligase CUL3. Chem Res Toxicol 21, 705-10 (2008).

25. Itoh, K. et al. An Nrf2/small Maf heterodimer mediates the induction of phase II detoxifying enzyme genes through antioxidant response elements. Biochem Biophys Res Commun 236, 313-22 (1997).

26. Moinova, H.R. \& Mulcahy, R.T. An electrophile responsive element (EpRE) regulates betanaphthoflavone induction of the human gamma-glutamylcysteine synthetase regulatory subunit gene. Constitutive expression is mediated by an adjacent AP-1 site. J Biol Chem 273, 14683-9 (1998).

27. Prommana, P. et al. Inducible knockdown of Plasmodium gene expression using the gImS ribozyme. PLoS One 8, e73783 (2013).

28. Naik, R.S., Krishnegowda, G. \& Gowda, D.C. Glucosamine inhibits inositol acylation of the glycosylphosphatidylinositol anchors in intraerythrocytic Plasmodium falciparum. J Biol Chem 278, 2036-42 (2003).

29. Mok, S. et al. Drug resistance. Population transcriptomics of human malaria parasites reveals the mechanism of artemisinin resistance. Science 347, 431-5 (2015).

30. Hott, A. et al. Artemisinin-resistant Plasmodium falciparum parasites exhibit altered patterns of development in infected erythrocytes. Antimicrob Agents Chemother 59, 3156-67 (2015). 
31. Ghorbal, M. et al. Genome editing in the human malaria parasite Plasmodium falciparum using the CRISPR-Cas9 system. Nat Biotechnol 32, 819-21 (2014).

32. Wezena, C.A., Krafczyk, J., Staudacher, V. \& Deponte, M. Growth inhibitory effects of standard pro- and antioxidants on the human malaria parasite Plasmodium falciparum. Exp Parasitol 180, 64-70 (2017).

33. Djuika, C.F., Staudacher, V., Sanchez, C.P., Lanzer, M. \& Deponte, M. Knockout of the peroxiredoxin 5 homologue PFAOP does not affect the artemisinin susceptibility of Plasmodium falciparum. Sci Rep 7, 4410 (2017).

34. Gao, L. et al. Novel n-3 fatty acid oxidation products activate Nrf2 by destabilizing the association between Keap1 and Cullin3. J Biol Chem 282, 2529-37 (2007).

35. Klayman, D.L. Qinghaosu (artemisinin): an antimalarial drug from China. Science 228, 1049-55 (1985).

36. Asawamahasakda, W., Ittarat, I., Pu, Y.M., Ziffer, H. \& Meshnick, S.R. Reaction of antimalarial endoperoxides with specific parasite proteins. Antimicrob Agents Chemother 38, 1854-8 (1994).

37. Robert, A., Benoit-Vical, F., Claparols, C. \& Meunier, B. The antimalarial drug artemisinin alkylates heme in infected mice. Proc Natl Acad Sci U S A 102, 13676-80 (2005).

38. Wang, J. et al. Haem-activated promiscuous targeting of artemisinin in Plasmodium falciparum. Nat Commun 6, 10111 (2015).

39. Ismail, H.M. et al. Artemisinin activity-based probes identify multiple molecular targets within the asexual stage of the malaria parasites Plasmodium falciparum 3D7. Proc Natl Acad Sci U S A 113, 2080-5 (2016).

40. Klonis, N. et al. Artemisinin activity against Plasmodium falciparum requires hemoglobin uptake and digestion. Proc Natl Acad Sci U S A 108, 11405-10 (2011).

41. Dinkova-Kostova, A.T. et al. Direct evidence that sulfhydryl groups of Keap1 are the sensors regulating induction of phase 2 enzymes that protect against carcinogens and oxidants. Proc Natl Acad Sci U S A 99, 11908-13 (2002).

42. Yamamoto, T. et al. Physiological significance of reactive cysteine residues of Keap1 in determining Nrf2 activity. Mol Cell Biol 28, 2758-70 (2008).

43. Kobayashi, M. et al. The antioxidant defense system Keap1-Nrf2 comprises a multiple sensing mechanism for responding to a wide range of chemical compounds. Mol Cell Biol 29, 493-502 (2009).

44. Siddiqui, G., Srivastava, A., Russell, A.S. \& Creek, D.J. Multi-omics Based Identification of Specific Biochemical Changes Associated With PfKelch13-Mutant Artemisinin-Resistant Plasmodium falciparum. J Infect Dis 215, 1435-1444 (2017).

45. Xie, S.C. et al. Haemoglobin degradation underpins the sensitivity of early ring stage Plasmodium falciparum to artemisinins. J Cell Sci 129, 406-16 (2016).

46. Turra, G.L., Schneider, L., Liedgens, L. \& Deponte, M. Testing the CRISPR-Cas9 and gImS ribozyme systems in Leishmania tarentolae. Mol Biochem Parasitol 241, 111336 (2021).

47. Scholz, J., Besir, H., Strasser, C. \& Suppmann, S. A new method to customize protein expression vectors for fast, efficient and background free parallel cloning. BMC Biotechnol 13, 12 (2013).

48. Trager, W. \& Jensen, J.B. Human malaria parasites in continuous culture. Science 193, 673-5 (1976).

49. Wezena, C.A. et al. The cytosolic glyoxalases of Plasmodium falciparum are dispensable during asexual blood-stage development. Microb Cell 5, 32-41 (2017).

50. Deitsch, K., Driskill, C. \& Wellems, T. Transformation of malaria parasites by the spontaneous uptake and expression of DNA from human erythrocytes. Nucleic Acids Res 29, 850-3 (2001).

51. Hasenkamp, S., Russell, K.T. \& Horrocks, P. Comparison of the absolute and relative efficiencies of electroporation-based transfection protocols for Plasmodium falciparum. Malar J 11, 210 (2012). 
52. Lambros, C. \& Vanderberg, J.P. Synchronization of Plasmodium falciparum erythrocytic stages in culture. J Parasitol 65, 418-20 (1979).

53. Seesui, K., Imtawil, K., Chanetmahun, P., Laummaunwai, P. \& Boonmars, T. An Alternative Method for Extracting Plasmodium DNA from EDTA Whole Blood for Malaria Diagnosis. Korean J Parasitol 56, 25-32 (2018).

54. Benting, J., Mattei, D. \& Lingelbach, K. Brefeldin A inhibits transport of the glycophorinbinding protein from Plasmodium falciparum into the host erythrocyte. Biochem J $\mathbf{3 0 0}$ ( Pt 3), 821-6 (1994).

55. Smilkstein, M., Sriwilaijaroen, N., Kelly, J.X., Wilairat, P. \& Riscoe, M. Simple and inexpensive fluorescence-based technique for high-throughput antimalarial drug screening. Antimicrob Agents Chemother 48, 1803-6 (2004).

56. Kavran, J.M. \& Leahy, D.J. Coupling antibody to cyanogen bromide-activated sepharose. Methods Enzymol 541, 27-34 (2014).

57. Ellman, G.L. A colorimetric method for determining low concentrations of mercaptans. Arch Biochem Biophys 74, 443-50 (1958).

58. Perez-Iratxeta, C. \& Andrade-Navarro, M.A. K2D2: estimation of protein secondary structure from circular dichroism spectra. BMC Struct Biol 8, 25 (2008). 


\section{Figure 1}

a

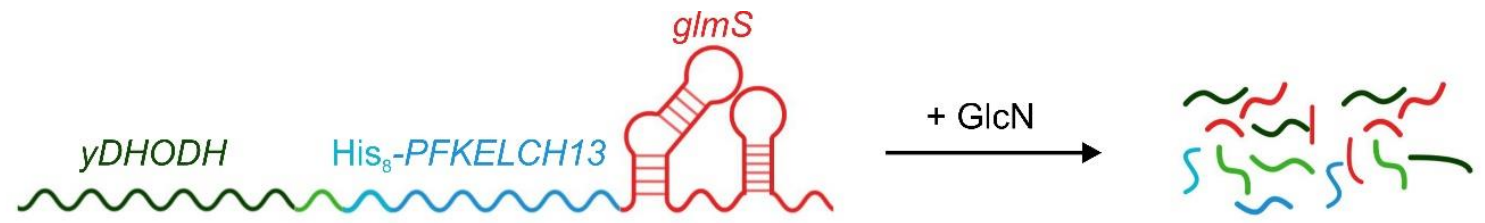

$2 \mathrm{~A}$

b
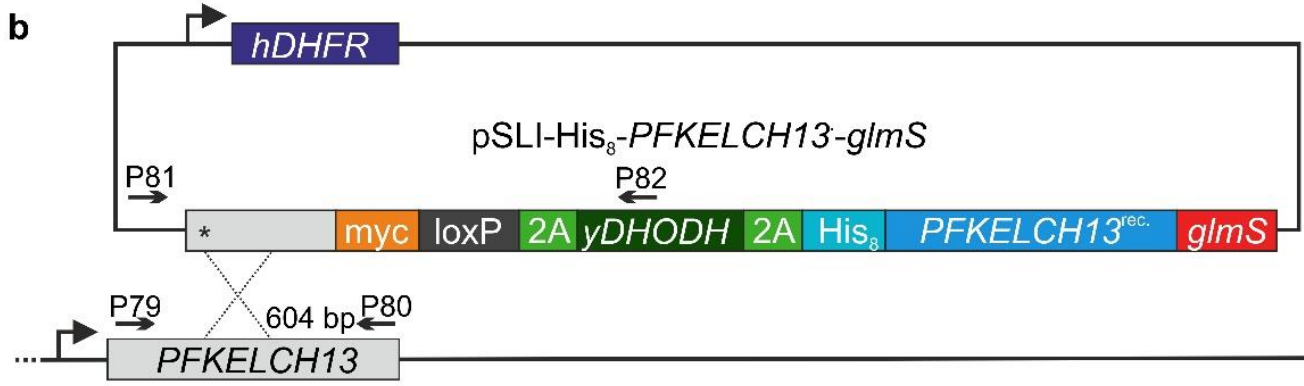

Chr. 13

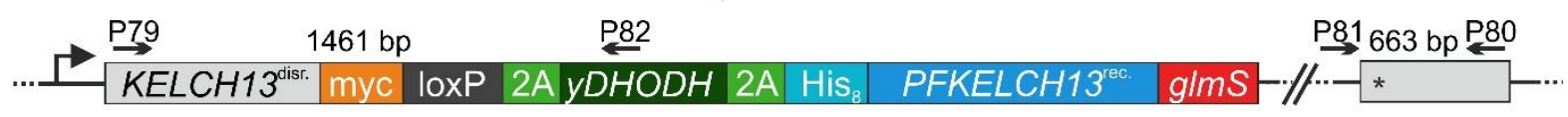

C

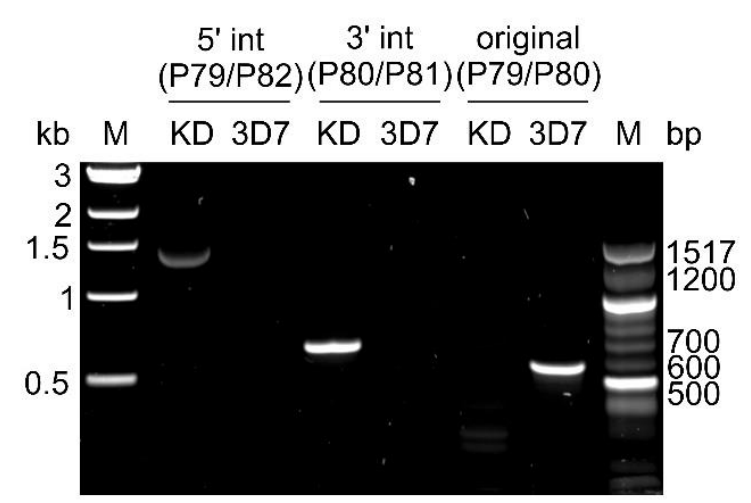

d

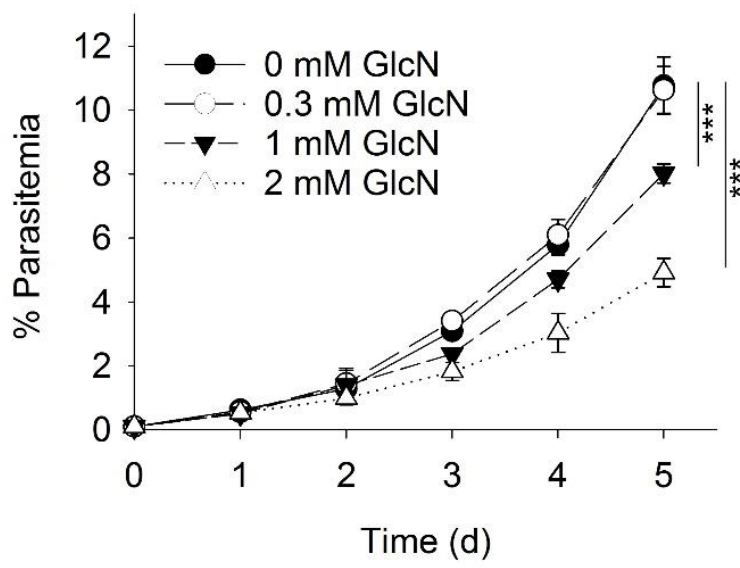

$\mathrm{His}_{8}$-PFKELCH13-M9

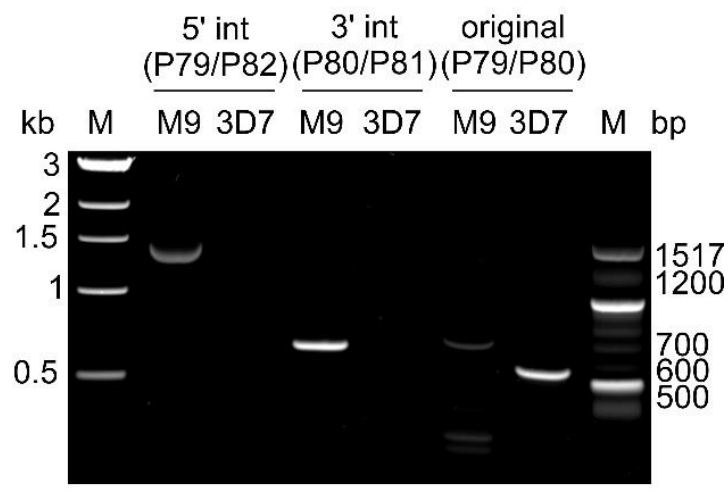

$\mathrm{His}_{8}-$ PFKELCH13-M9

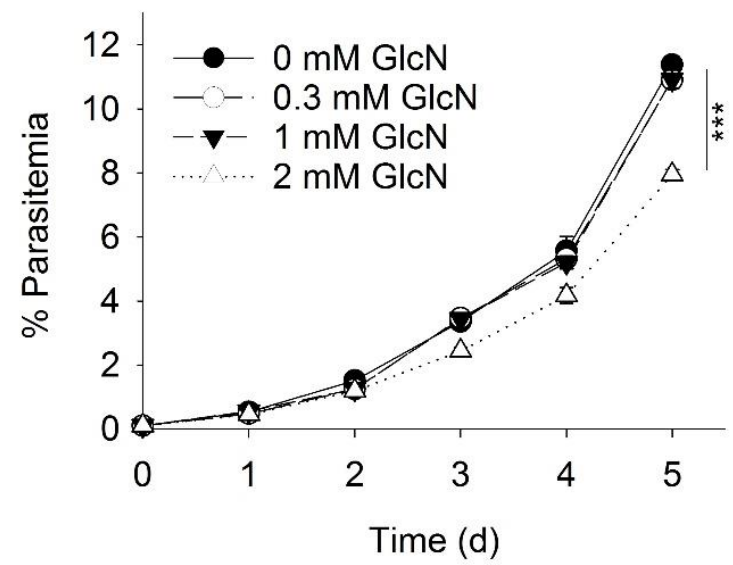




\section{Figure 1 | Generation and validation of a regulatable PFKELCH13 knockdown strain.}

(a) Schematic overview of the knockdown strategy. The addition of glucosamine (GlcN) results in the activation of the glmS ribozyme and the degradation of mRNA encoding cytosolic yeast dihydroorotate dehydrogenase $(y D H O D H)$, the self-cleaving 2A peptide (2A) and Hiss-tagged Kelch13. (b) Schematic overview of the gene replacement by selection-linked integration (SLI). Plasmid-containing 3D7 parasites with human dihydrofolate reductase (hDHFR) are first selected using the antifolate WR99120. Disruption of the endogenous PFKELCH13 openreading frame by homologous recombination provides the promoter for the fusion construct between recodonized PFKELCH13 ${ }^{\text {rec }}$ and $y D H O D H$, the resistance marker against DSM1. Primer positions and expected product sizes for PCR analysis are highlighted. (c) PCR analyses using the indicated primer pairs from panel b confirmed the successful integration for knockdown strain His8-PFKELCH13-gImS (KD) and strain His-PFKELCH13-M9 (M9) with active and inactive glmS, respectively. Genomic DNA from parental strain 3D7 served as a control. (d) Growth curve analyses for ansynchronous blood-stage cultures of strain His8PFKELCH13-glmS in the presence of different concentrations of GlcN. The parasitemia was determined from Giemsa-stained blood smears. Strain His - PFKELCH13-M9 served as a negative control to discriminate between growth defects that were caused by the knockdown of $K E L C H 13$ or the toxicity of GICN. All data points represent the mean \pm standard deviation of three independent experiments. Statistical analyses were performed using the one-way ANOVA method in SigmaPlot13 $\left(\mathrm{P} \leq 0.001:^{* * *}\right)$. Source data are provided as a Source Data file. 


\section{Figure 2}

a $\mathrm{His}_{8}-$ PFKELCH13-gimS

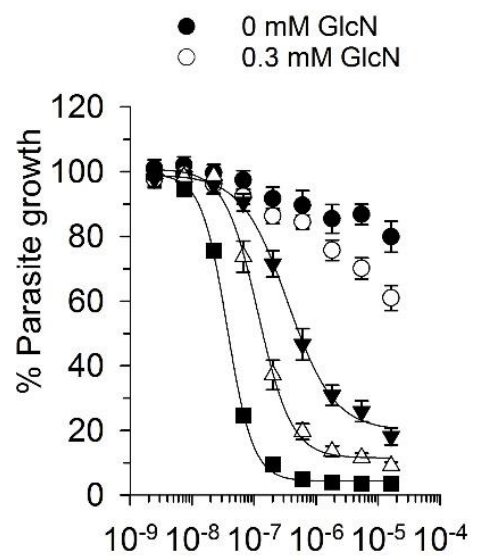

[DSM1] (M)
$\mathrm{His}_{8}-$ PFKELCH13-M9

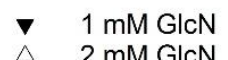

- 3D7wt

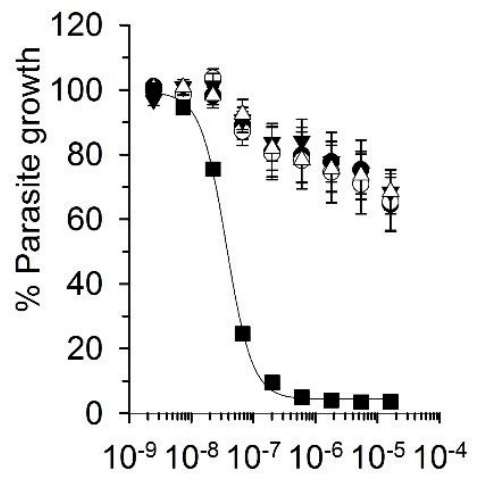

[DSM1] (M)
C

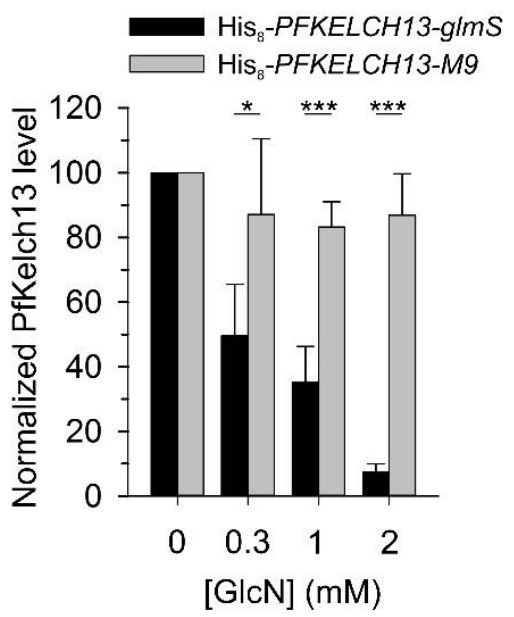

b

$\mathrm{His}_{8}$-PFKELCH13-gimS

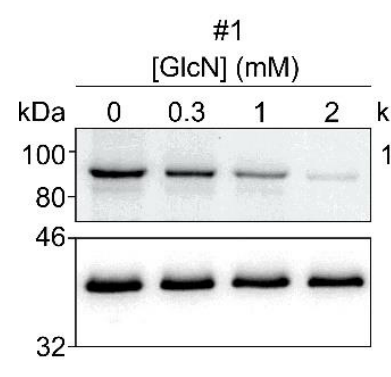

\#1

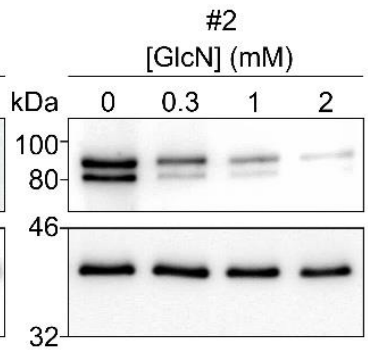

\#3

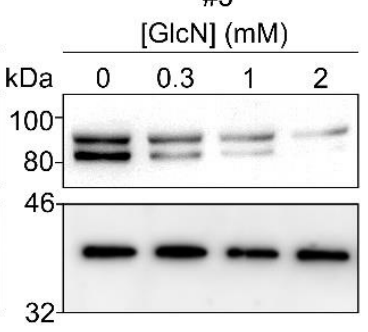

$\mathrm{His}_{8}-$ PFKELCH13-M9

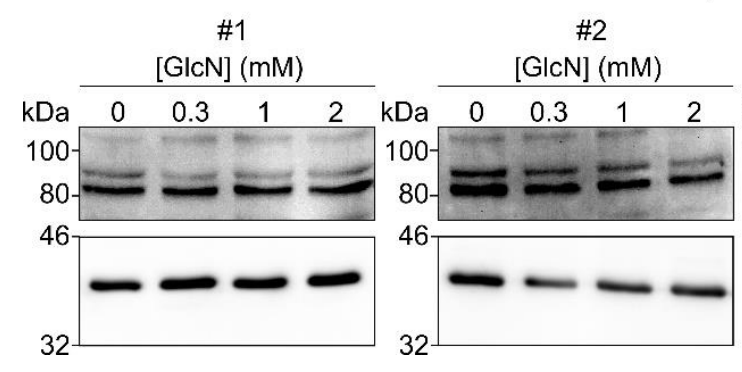

\#3

\#4

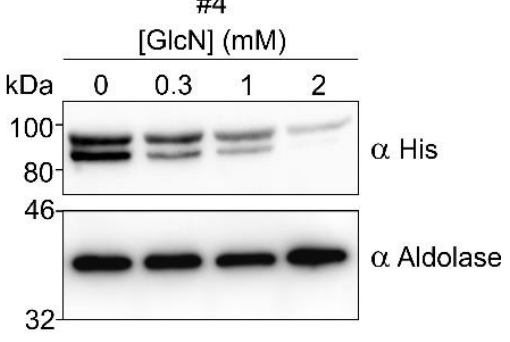

\#4

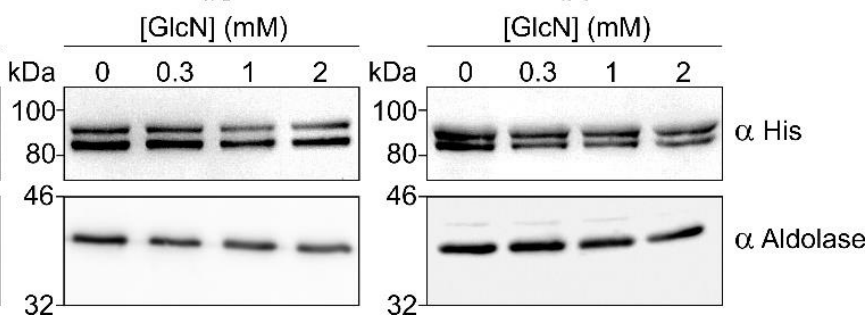

Figure 2 | Quantification of the down-regulation of His8-PfKelch13. (a) $\mathrm{EC}_{50}$ values for DSM1 were determined in 96-well plates using a SYBR green assay. Ring-stage parasites from strains $\mathrm{His}_{8}$-PFKELCH13-gImS and $\mathrm{His}_{8}$-PFKELCH13-M9 were incubated in 96-well plates for $140 \mathrm{~h}$ with or without $0.3 \mathrm{mM}$ GIcN and $2.5 \mathrm{nM}-16.2 \mu \mathrm{M}$ DSM1. Strain 3D7 served as a positive control. All data points represent the mean \pm standard deviation of four independent experiments with triplicate measurements. (b) Ring-stage parasites from strains $\mathrm{His}_{8}-$ PFKELCH13-gImS and His - PFKELCH13-M9 were incubated for two cycles with the indicated concentrations of GlcN before western blot analysis against the His-tag. The 
calculated molecular mass of $\mathrm{His}_{8}$-PfKelch13 is $85.2 \mathrm{kDa}$. Aldolase was used as a loading control for normalization. (c) Quantification of the normalized western blot signals from the four independent experiments in panel b. Statistical analyses were performed using the one-way ANOVA method in SigmaPlot13 ( $\left.P<0.05:{ }^{*} ; \mathrm{P} \leq 0.001:{ }^{* * *}\right)$. Source data are provided as a Source Data file. 


\section{Figure 3}
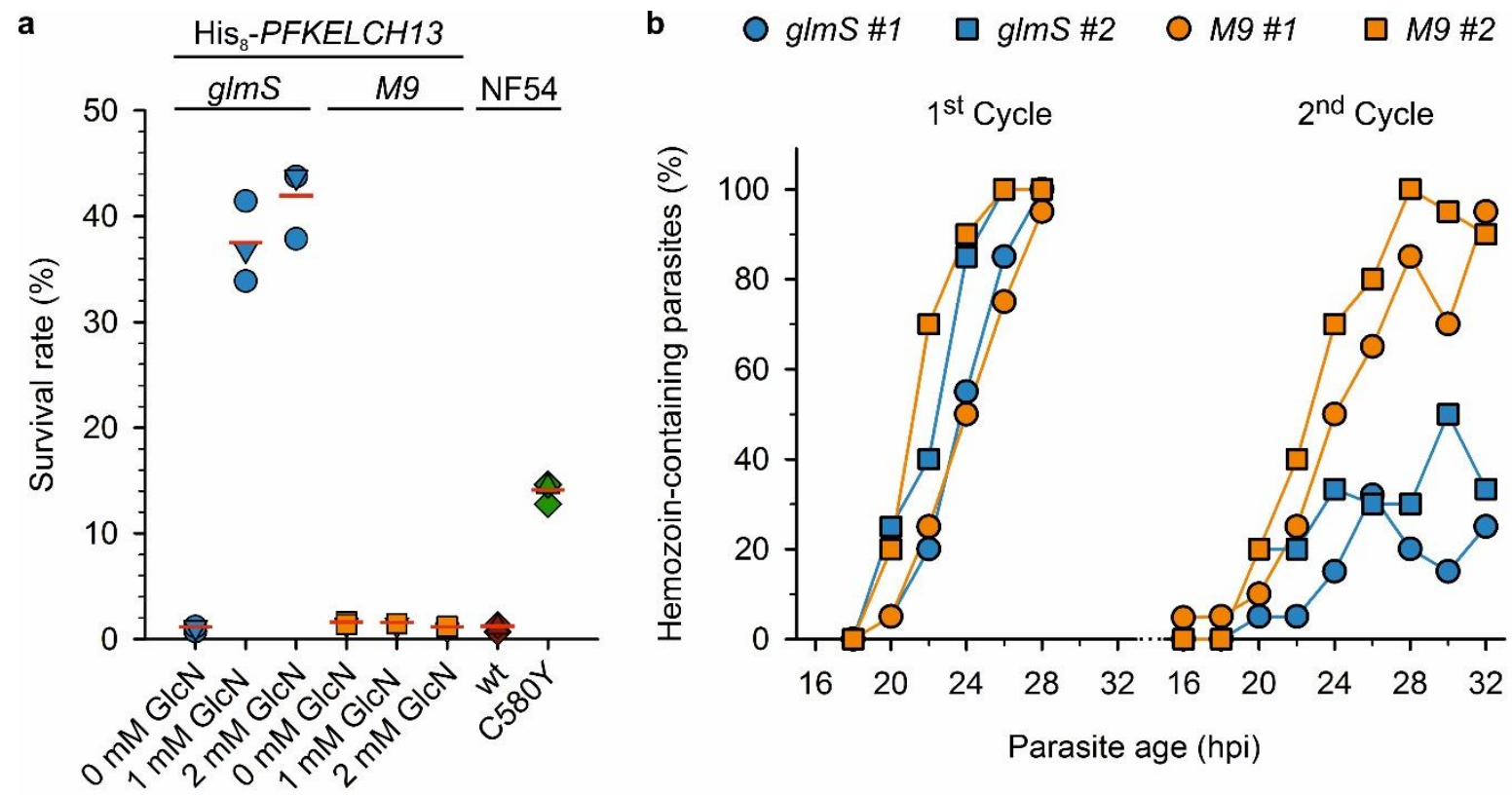

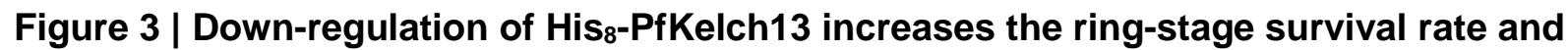
impairs hemozoin formation. (a) Ring-stage His ${ }_{8}-$ PFKELCH13-glmS parasites ( $\left.g / m S\right)$ were pretreated for two cycles with or without the indicated GlcN concentration. RSAs were subsequently performed with $0.7 \mu \mathrm{M}$ artesunate without GlcN. The RSAs were validated using strain NF54K13 ${ }^{\mathrm{C} 580 \mathrm{Y}}$ as a positive control. Strains $\mathrm{His}_{8}-\mathrm{PFKELCH13-M9}$ (M9) and wild-type NF54 (wt) served as negative controls. Each symbol represents a data point from one of three independent experiments. (b) Phenotypical analysis of His ${ }_{8}$ PFKELCH13-glmS knockdown

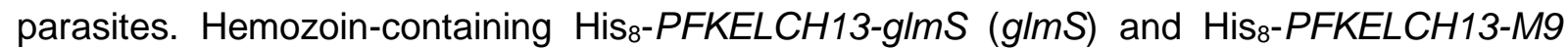
(M9) parasites that were treated with $1 \mathrm{mM} \mathrm{GlcN}$ were quantified during the first and second cycle 16-32 hpi. The percentage was determined by light microscopy of stained thin blood smears. Representative parasites are shown in Fig. S1. All data points represent the individual data points of two independent biological replicates. Source data are provided as a Source Data file. 


\section{Figure 4}

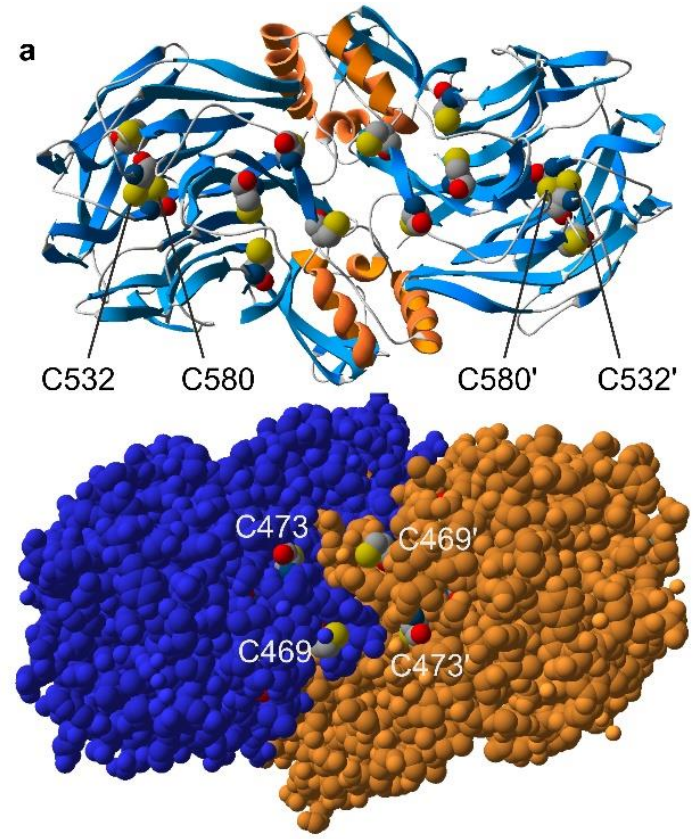

b
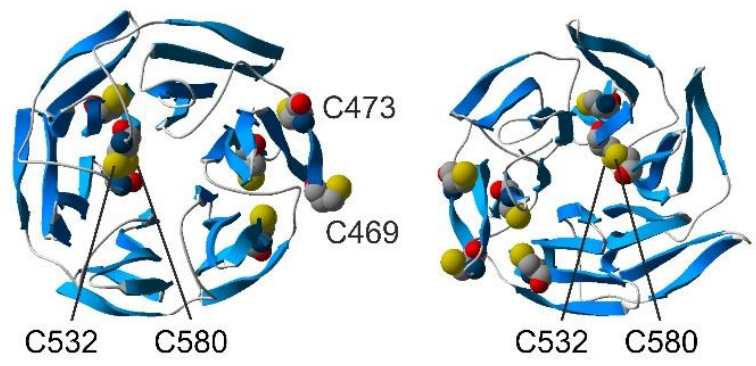

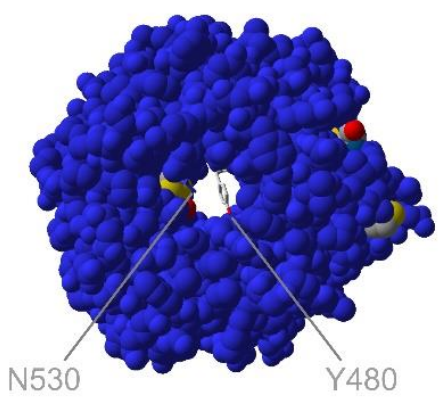

Top view

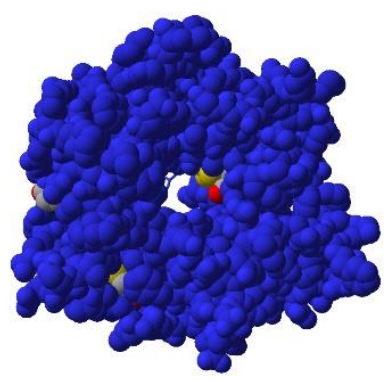

Bottom view

c

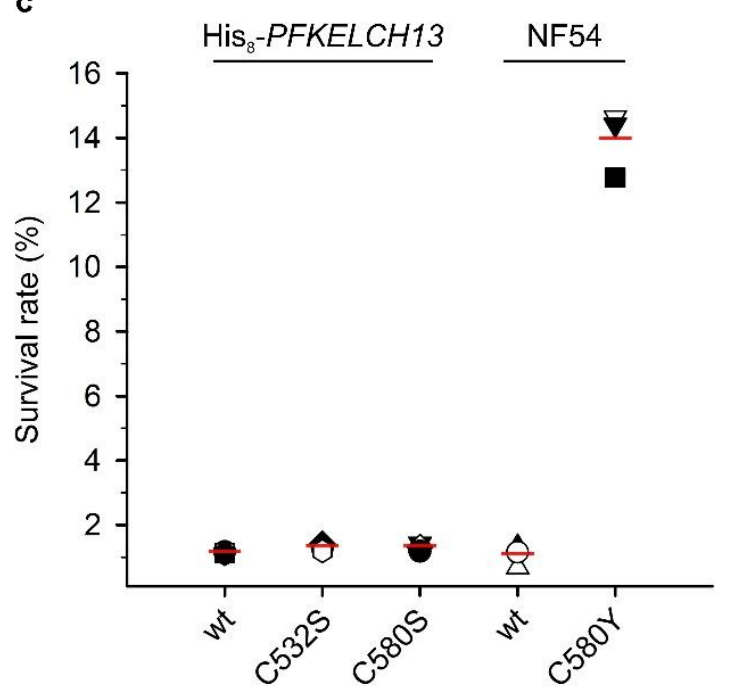

d

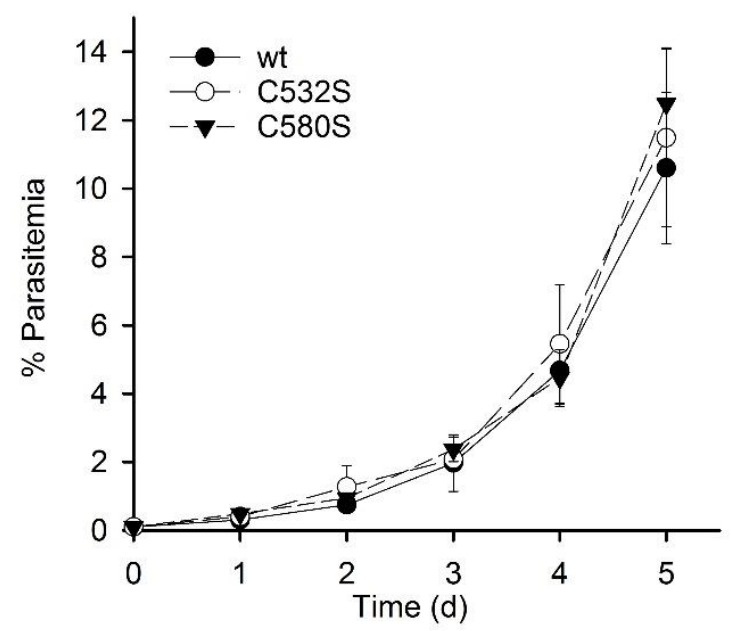

Figure 4 | Physiological relevance of the thiol groups of C532 and C580. (a) Overview of the positions and accessibility of the seven cysteine residues in crystallized recombinant PfKelch ${ }^{350-726}$, comprising the BTB domain and the Kelch domain (PDB entry 4YY8). The two subunits of the homodimer are coloured in blue and orange in the space-filling model at the bottom. (b) Top and bottom view of the Kelch domain. Residues C532 and C580 can form a disulfide bond that faces the pore of the $\beta$-propeller. Residues N530 and the gatekeeper residue Y480 partially block the access of the pore from the top and are shown in stick representation. (c) RSAs with $0.7 \mu \mathrm{M}$ artesunate for strains $\mathrm{His}_{8}-P F K E L C H 13^{\text {wt }}$ (wt), His ${ }^{-}$ 
PFKELCH13 ${ }^{\mathrm{C} 532 \mathrm{~S}}$ (C532S), and His 8 -PFKELCH13 ${ }^{\mathrm{C} 580 \mathrm{~S}}$ (C580S). Strains NF54K13 $13^{\mathrm{C} 580 \mathrm{Y}}$ (C580Y) and wild-type NF54 (wt) served as controls. Each symbol represents a data point from one of three independent experiments. (d) Growth curve analyses for ansynchronous bloodstage cultures with Hiss-tagged PfKelch13 cysteine mutants. The parasitemia for the indicated mutant strains was determined from Giemsa-stained blood smears. Strain His ${ }_{8}-P F K E L C H 13^{\text {wt }}$ served as a control. All data points represent the mean \pm standard deviation of three independent experiments. Statistical analyses were performed using the one-way ANOVA method in SigmaPlot13 $\left(P \leq 0.001:{ }^{* * *}\right)$. Source data are provided as a Source Data file. 


\section{Figure 5}

a

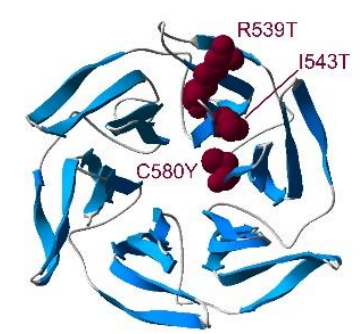

c

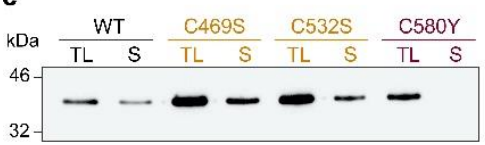

$\mathrm{kDa} \frac{\mathrm{C} 542 \mathrm{~S}}{\mathrm{TLS}} \frac{\mathrm{C} 580 \mathrm{~S}}{\mathrm{TLS} \mathrm{S}} \frac{\mathrm{C} 580 \mathrm{~F}}{\mathrm{TLS} \mathrm{S}} \frac{\mathrm{C} 696 \mathrm{~S}}{\mathrm{TL} \mathrm{S}}$

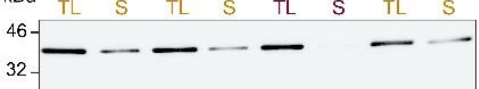

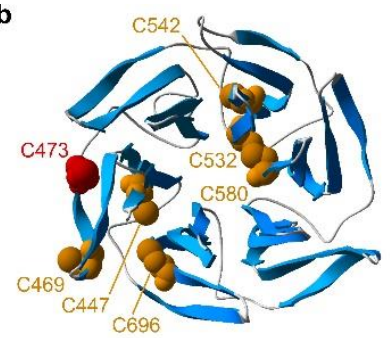
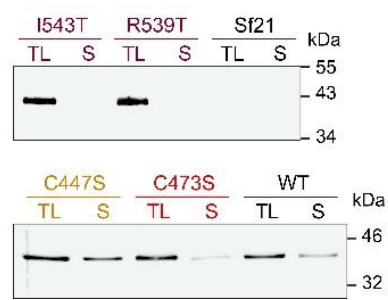

d
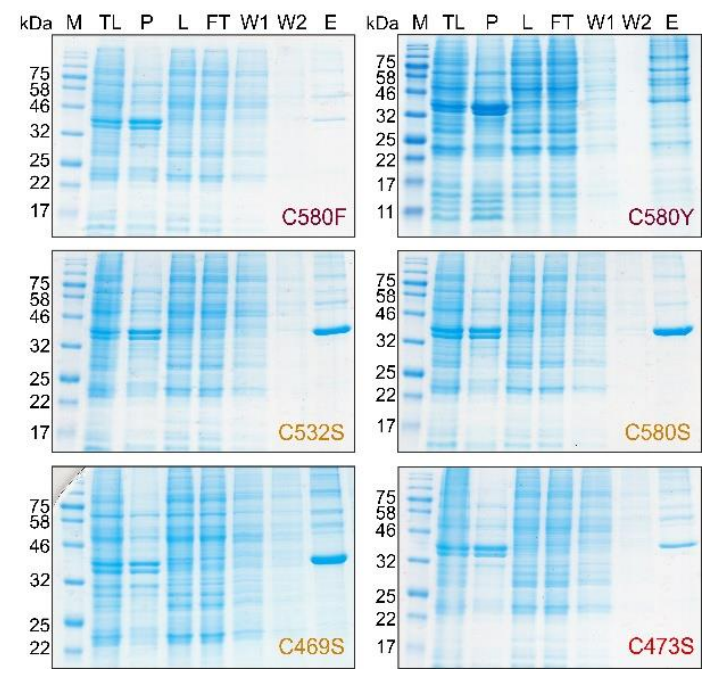

Figure 5 | Solubility of recombinant PfKelch13 $137-726$ mutants. (a) Overview of three residues in the $\beta$-propeller of PfKelch13 (PDB entry 4YY8) that are frequently mutated in $P$. falciparum field isolates and that are linked to a decreased artemisinin susceptibility. (b) Overview of the seven cysteine residues in the $\beta$-propeller of PfKelch13 including disulfidebridged C532 and C580 (PDB entry 4YY8). (c) Solubility screen of wild-type (WT) and mutant $\mathrm{MH}_{8}$ SR-tagged PfKelch13 $3^{337-726}$ in Sf21 cells. Cells were lysed by three freeze-thaw cycles and equal volumes of the total lysate (TL) and the soluble fraction (S) were separated by SDS PAGE and analyzed by western blotting against the His-tag. Lysates from SF21 cells without PfKelch13 ${ }^{337-726}$ served as a negative control. d) Representative purifications of mutant PfKelch13337-726 from panel c. Cells were lysed by three freeze-thaw cycles. The total lysate $(T L)$ was centrifuged, yielding insoluble pellet $(P)$ and supernatant $(L)$, which was loaded on a Ni-NTA agarose column. The flow-through (FT) was discarded, the resin was washed (W) and the bound protein eluted $(\mathrm{E})$ with imidazole. 


\section{Figure 6}

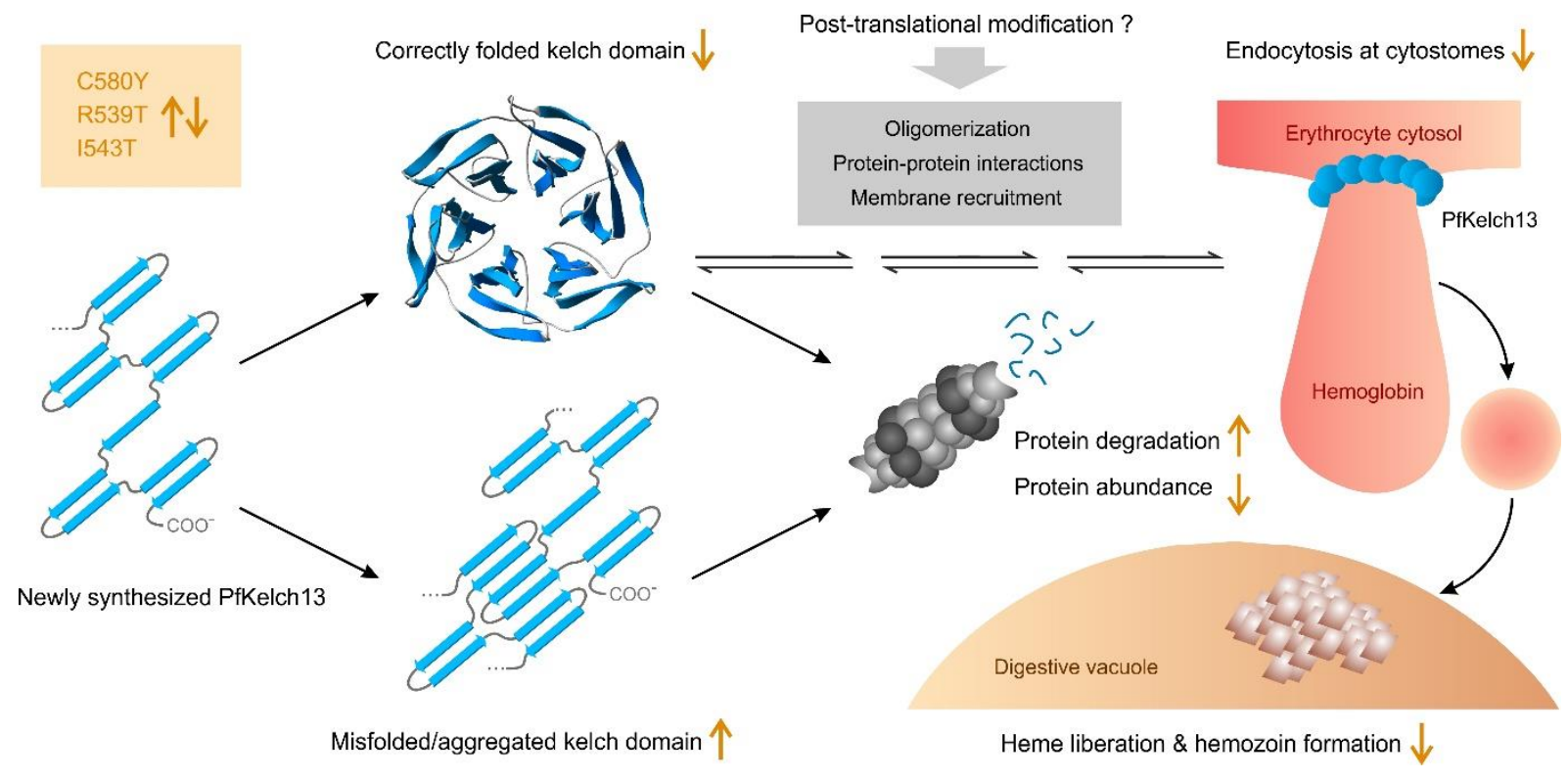

Figure 6 | Updated model for the decreased artemisinin susceptibility of strains with mutant or down-regulated PfKelch13. Common field mutations destabilize the kelch domain, resulting in a decreased protein abundance, endocytosis, and liberation of heme, which activates artemisinin. This phenotype can be mimicked in parasites with down-regulated PfKelch13. 


\section{Supplementary Information}

\section{Protein abundance and folding rather than the redox state of Kelch13 determine the artemisinin susceptibility of Plasmodium falciparum}

Robin Schumann', Eileen Bischoff', Severina Klaus², Sophie Möhring ${ }^{1}$, Julia Flock ${ }^{3}$, Sandro Keller ${ }^{4-7}$, Kim Remans $^{3}$, Markus Ganter ${ }^{2}$ \& Marcel Deponte ${ }^{1 *}$

${ }^{1}$ Faculty of Chemistry, Comparative Biochemistry, Technische Universität Kaiserslautern, D67663 Kaiserslautern, Germany

${ }^{2}$ Centre for Infectious Diseases, Parasitology, Heidelberg University Hospital, D-69120 Heidelberg, Germany

${ }^{3}$ Protein Expression and Purification Core Facility, European Molecular Biology Laboratory (EMBL), D-69117 Heidelberg, Germany

${ }^{4}$ Molecular Biophysics, Technische Universität Kaiserslautern, D-67663 Kaiserslautern, Germany

${ }^{5}$ Biophysics, Institute of Molecular Biosciences (IMB), NAWI Graz, University of Graz, Humboldtstr. 50/III, 8010 Graz, Austria

${ }^{6}$ Field of Excellence BioHealth, University of Graz, Graz, Austria

${ }^{7}$ BioTechMed-Graz, Graz, Austria

${ }^{*}$ Correspondence and requests for materials should be addressed to M.D. (deponte@chemie.uni-kl.de) 


\section{Figure S1}

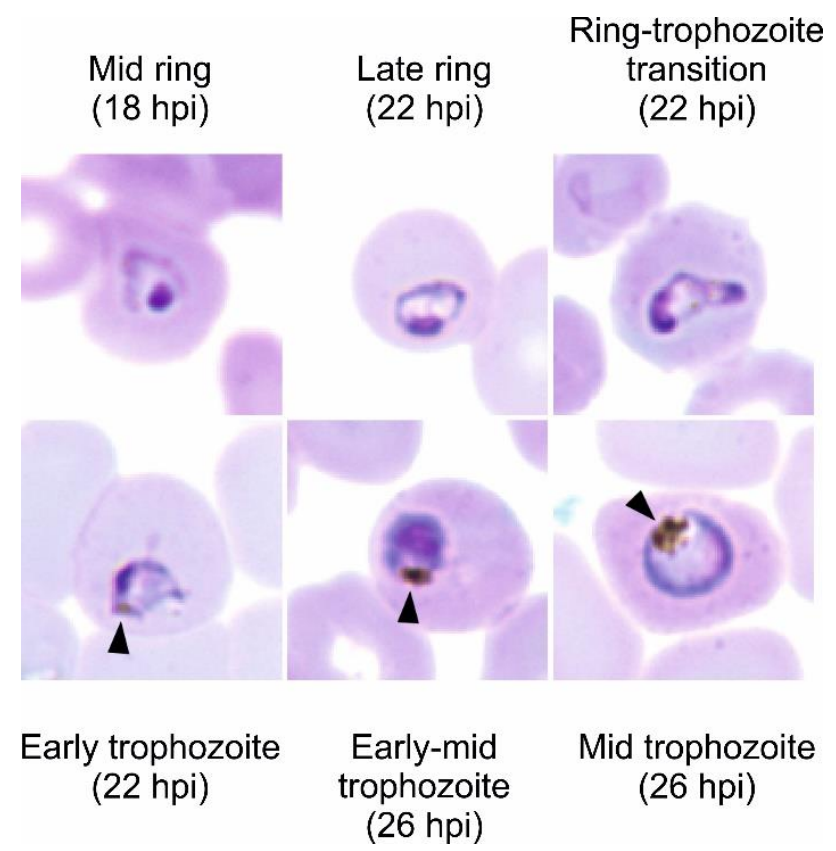

Supplementary Figure 1 | Representative images of stained blood-stage parasites. The hemozoin content of synchronized His ${ }_{8}$ PFKELCH13-glmS and His ${ }_{8}$ PFKELCH13-M9 parasites was analyzed for air-dried and methanol-fixed thin blood smears that were stained with Hemacolor Rapid staining of blood smear solution. Images were acquired on a BZ-X800 microscope using the $60 \times$ oil objective. Time points when the cells were fixed are indicated. Visible hemozoin is labelled with an arrowhead. 


\section{Figure S2}
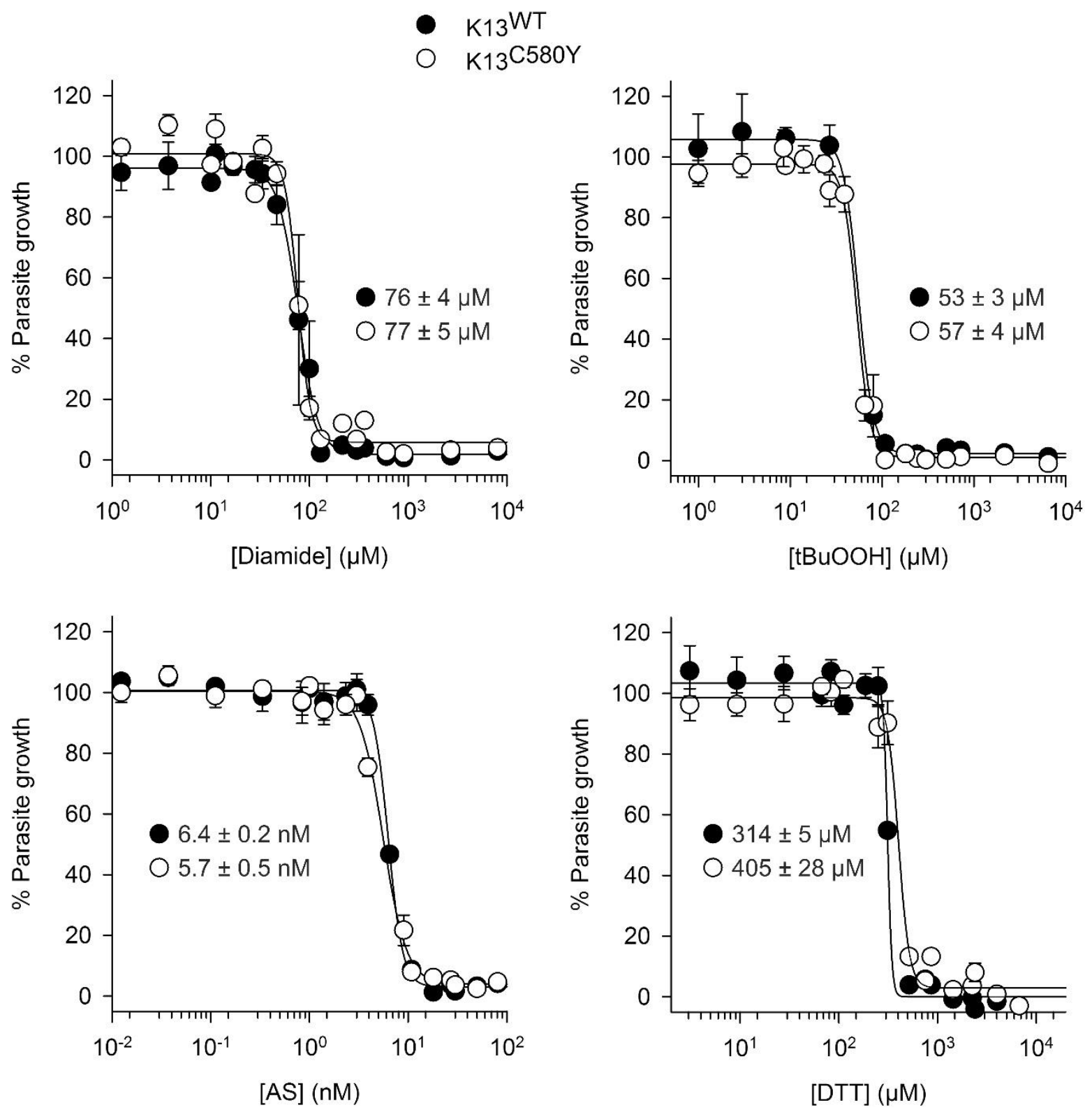

\section{Supplementary Figure 2 | Growth inhibitory effects of redox agents on strain}

NF54K13 ${ }^{\text {C580Y }}$. IC 50 values were determined in 96-well plates using a SYBR green assay for bolus treatments of ring stage parasites with the indicated concentrations of the disulfideinducer diamide, the oxidant $\mathrm{tBuOOH}$, the endoperoxide artesunate (AS) and the reducing agent DTT. Each curve represents the mean \pm standard deviation of three independent experiments with triplicate measurements. Data were fitted and analyzed in SigmaPlot13 revealing no significant differences between the indicated $\mathrm{IC}_{50}$ values for strains $\mathrm{NF} 54 \mathrm{~K} 13^{\mathrm{C} 580 \mathrm{Y}}$ $\left(\mathrm{K} 13^{\mathrm{C} 580 \mathrm{Y}}\right)$ and wild-type NF54 $\left(\mathrm{K} 13^{\mathrm{WT}}\right)$. Source data are provided as a Source Data file. 


\section{Figure S3}

a
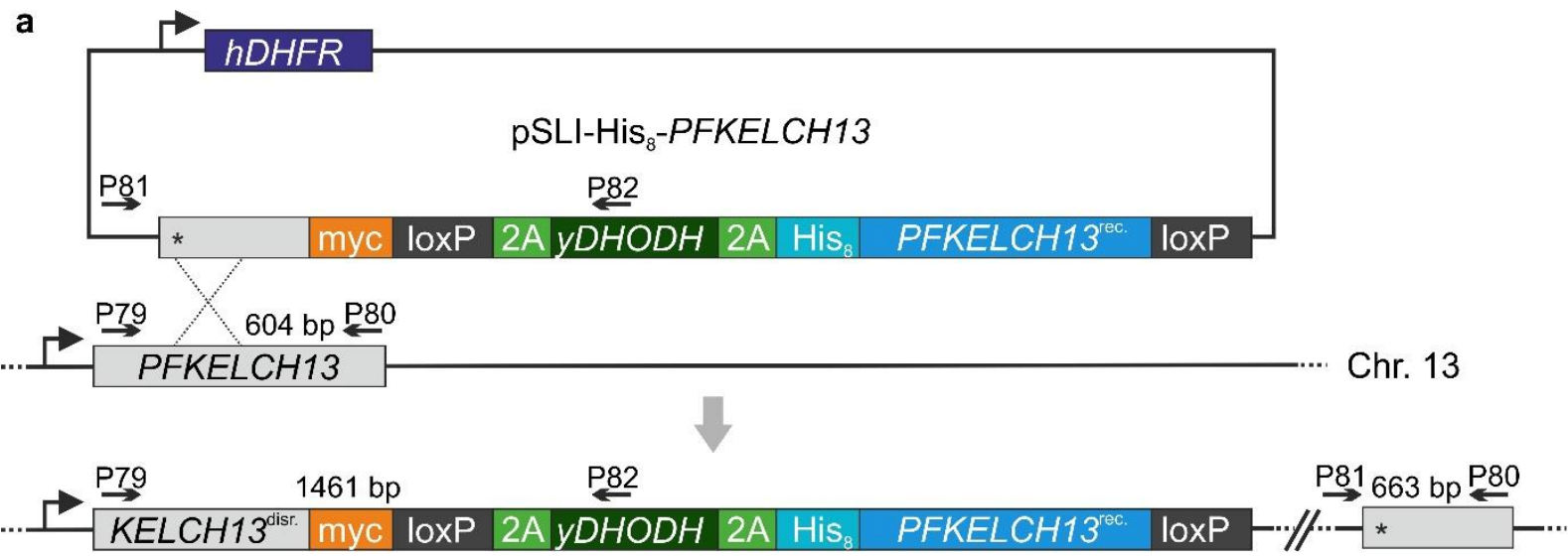

b

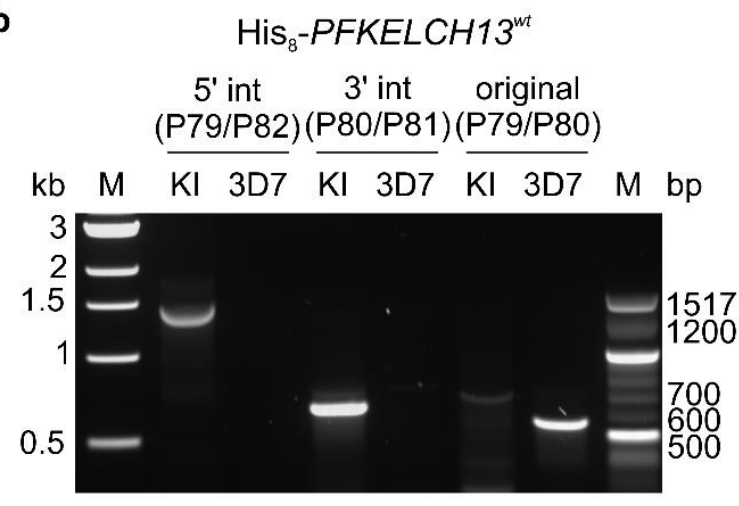

$\mathrm{His}_{8}$-PFKELCH13 ${ }^{\mathrm{C} 800 \mathrm{~S}}$

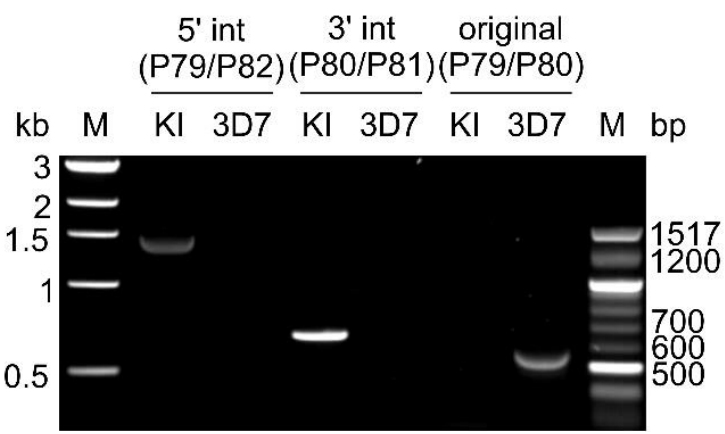

$\mathrm{His}_{8}-$ PFKELCH13 $3^{\mathrm{C} 532 \mathrm{~S}}$

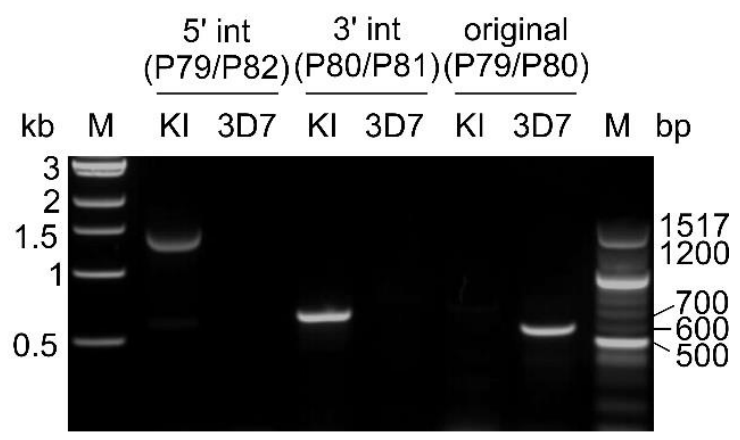

$\mathrm{His}_{8}-$ PFKELCH13 $3^{\mathrm{C} 469 \mathrm{~S}}$

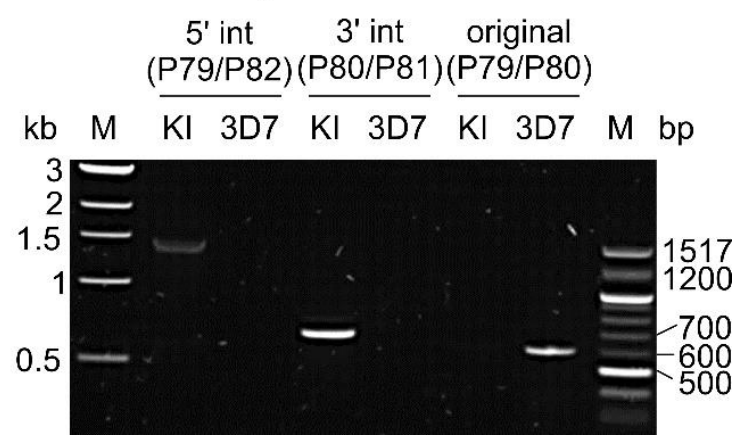

\section{Supplementary Figure 3 | Generation and validation of PFKELCH13 cysteine mutants.}

(a) Schematic overview of the SLI strategy. Recodonized PFKELCH13 encoding His8-tagged wild-type protein or a cysteine mutant was fused with the selection marker $y D H O D H$ and integrated into PFKELCH13 wild-type locus. Primer positions and expected product sizes for PCR analysis are highlighted. (b) PCR analyses using the indicated primer pairs from panel a confirmed the successful integration for wild-type strain His ${ }_{8}-P F K E L C H 13^{\text {wt }}$ and mutant strains $\mathrm{His}_{8}-$ PFKELCH13 ${ }^{\mathrm{C} 532 S}$, His $8_{8}-P F K E L C H 13^{C 580 S}$, and His ${ }_{8}-P F K E L C H 13^{C 469 S}$. Genomic DNA from parental strain 3D7 served as a control. 


\section{Figure S4}
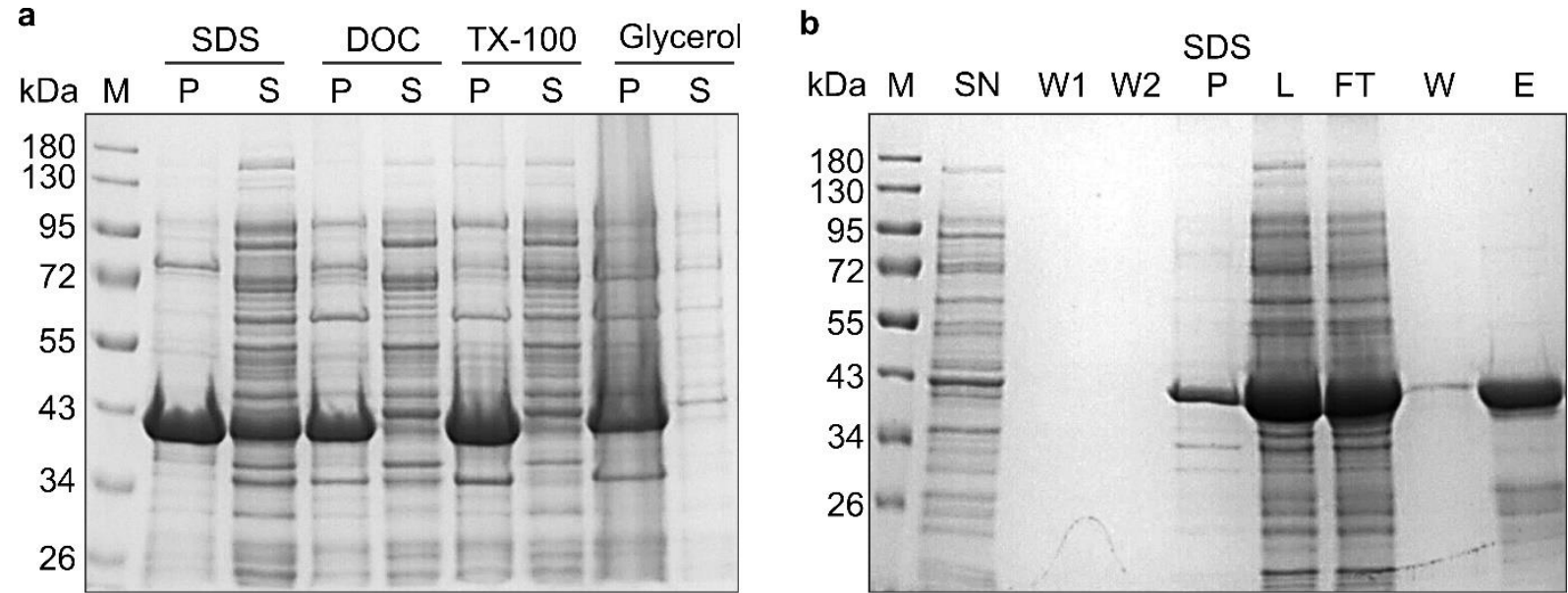

C
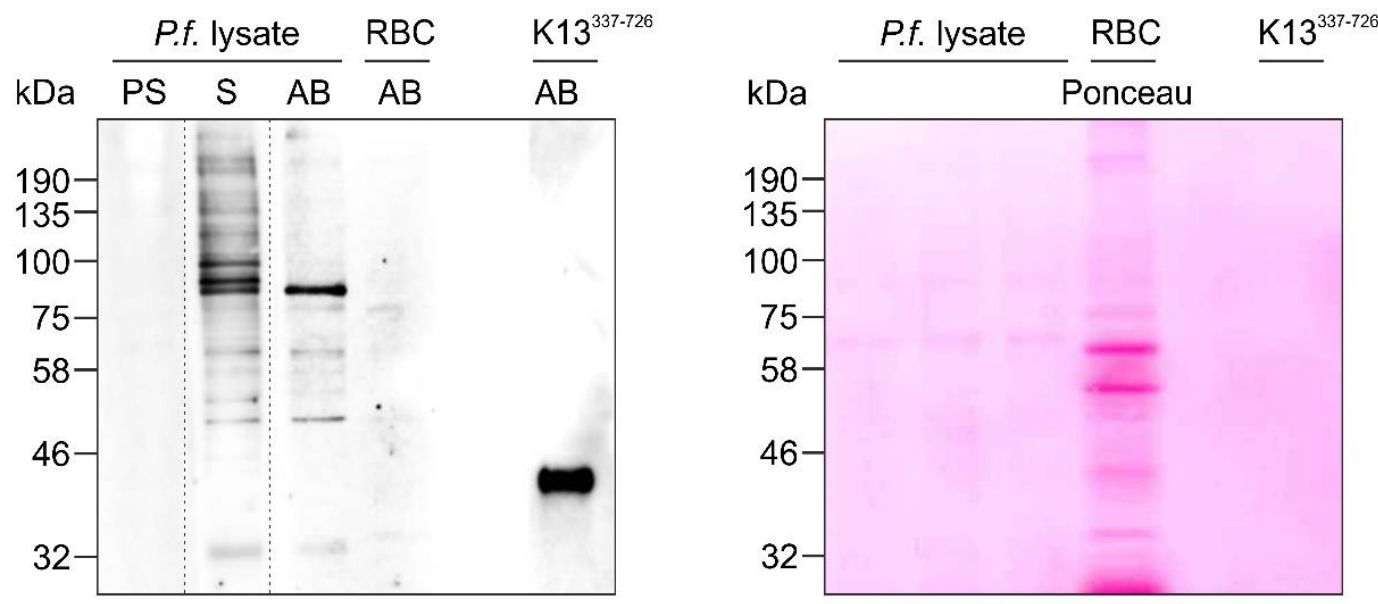

d 3D7

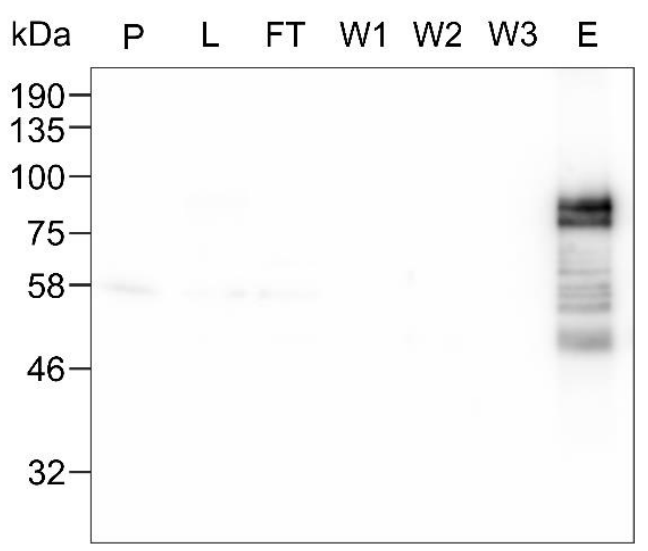

a PfKelch13

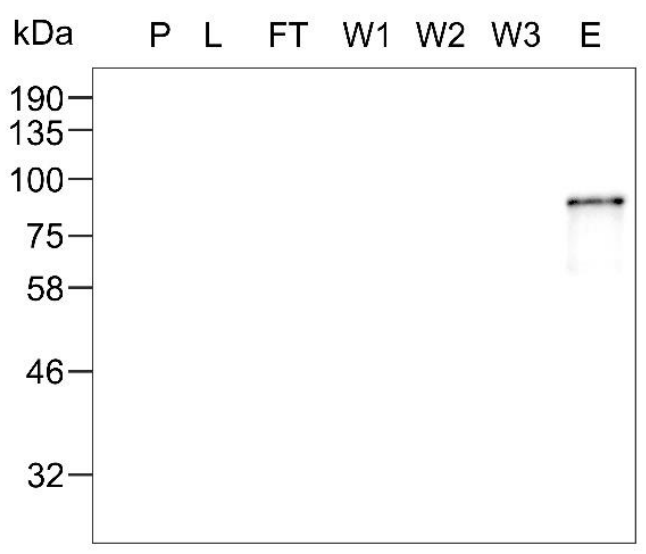

$\alpha$ His

Supplementary Figure 4 | Generation of an antibody and pull-down of PfKelch13.

(a) Solubilization screen for recombinant $\mathrm{MAH}_{6} \mathrm{VGT}$-tagged PfKelch13336-726 from E. coli. PfKelch $13^{336-726}$ was efficiently solubilized from washed inclusion bodies with solubilization 
buffer containing $2 \%$ SDS, whereas $2 \%$ deoxycholic acid (DOC), $2 \%$ Triton X-100 (TX-100) or 50\% glycerol did not show a solubilization effect. P: pellet, S: supernatant. (b) Purification of denatured recombinant PfKelch13 ${ }^{336-726}$. Inclusion bodies containing PfKelch13 ${ }^{336-726}$ were washed twice (W1, W2) and solubilized with $2 \%$ SDS. Excess SDS was precipitated on ice (SDS P). The supernatant (L) was loaded on a Ni-NTA agarose column. After washing (W), the protein was eluted with $200 \mathrm{mM}$ imidazole (E) and used for immunization, yielding the serum and antibody in panel c. SN: supernatant from the E. coli lysate, FT: flow through. (c) Purification and validation of a rabbit PfKelch13 antibody by western blot analysis. Left: Serum (S) as well as purified antibody $(A B)$ detected a protein with a molecular mass of $\sim 85 \mathrm{kDa}$ in a P. falciparum lysate. Endogenous PfKelch13 has a calculated molecular mass of $83.7 \mathrm{kDa}$. Decoration with preserum (PS) served as a negative control. No signal was detected in uninfected red blood cells (RBC). Recombinant PfKelch13 $337-726$ (K13 $\left.{ }^{337-726}\right)$ with a calculated molecular mass of $45.7 \mathrm{kDa}$ served as a positive control. Lanes contained either proteins from $3 \times 10^{7}$ purified trophozoites, $3 \times 10^{7}$ uninfected RBC or $10 \mathrm{ng}$ PfKelch13 $3^{336-726}$ as indicated. The dashed line indicates the cut membrane. Right: Ponceau S-staining of the membrane served as a loading control. (d) Purification of PfKelch13 from strain 3D7 (left) or His - PFKELCH13 ${ }^{\text {wt }}$ (right) by immunoaffinity chromatography. The PfKelch13 antibody from panel c was coupled to $\mathrm{CNBr}$-activated sepharose. Lysates with denatured proteins were prepared from $6 \times 10^{9}$ trophozoites. P, pellet; L, $0.2 \%$ of the loaded lysate; FT, flow-through; W1-3, wash fractions 13; E, $6.7 \%$ of the eluate. The purified proteins were detected using either the PfKelch13 rabbit antibody or a commercial anti-His 6 mouse antibody. 


\section{Figure S5}

a

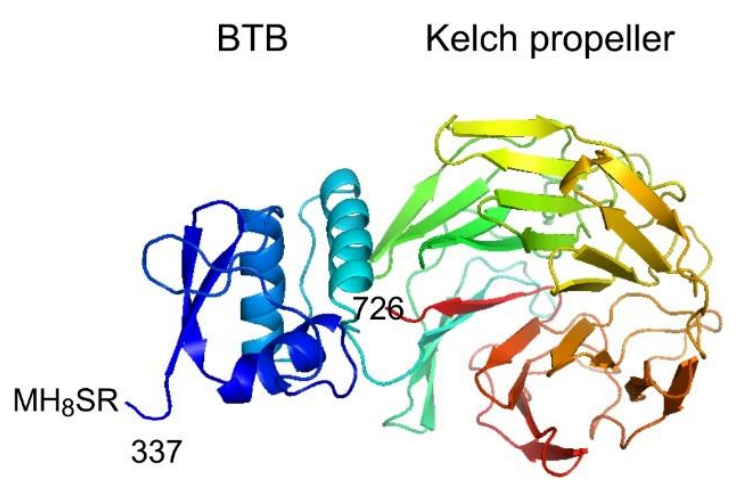

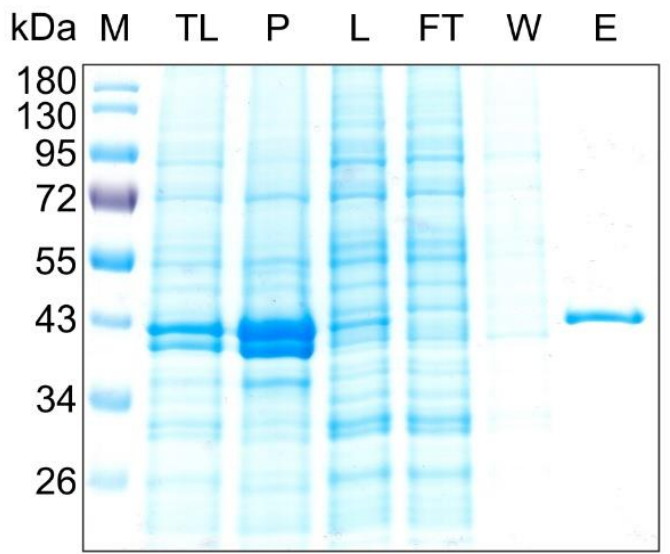

b

MHHHHHHHHSRDKKKIVDANIATETMIDINVGGAIFETSRHTLTQQKDSFIEKLLSGRHHVTRDKQGRI FLDRDSELFRIILNFLRNPLTIPIPKDLSESEALLKEAEFYGIKFLPFPLVFC I GGF DGVEYLNSMELL DISQQCWRMCTPMSTKKAYFGSAVLNNFLYVFGGNNYDYKALFETEVYDRLRDVWYVSSNLNIPRRNNC GVTSNGRIYCIGGYDGSSII PNVEAYDHRMKAWVEVAPLNTPRSSAMCVAFDNKIYVIGGTNGERLNSI EVYEEKMNKWEQFPYALLEARSSGAAFNYLNQIYVVGGIDNEHNILDSVEQYQPFNKRWQFLNGVPEKK MNFGAATLSDSY I ITGGENGEVLNSCHFFSPDTNEWQLGPSLLVPRFGHSVLIANI *

c

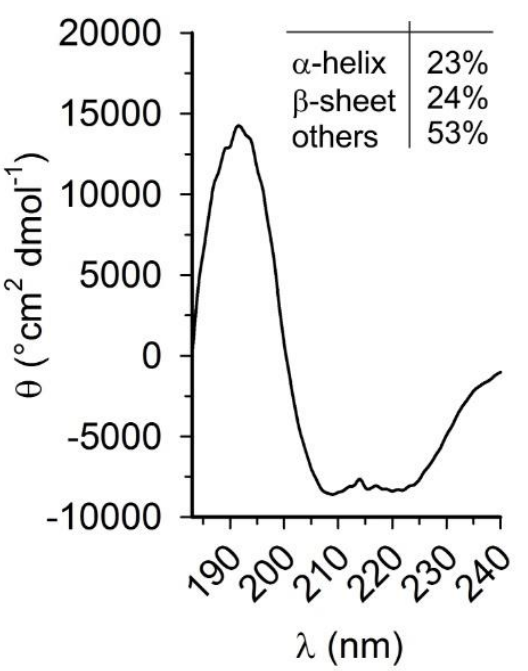

d

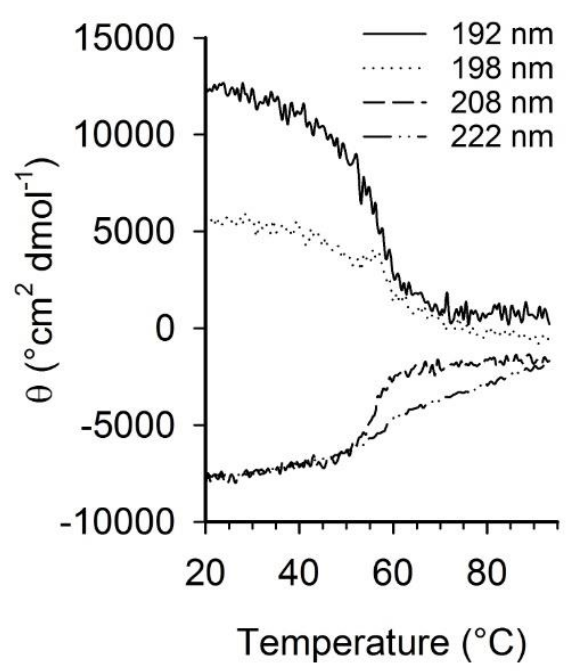

e

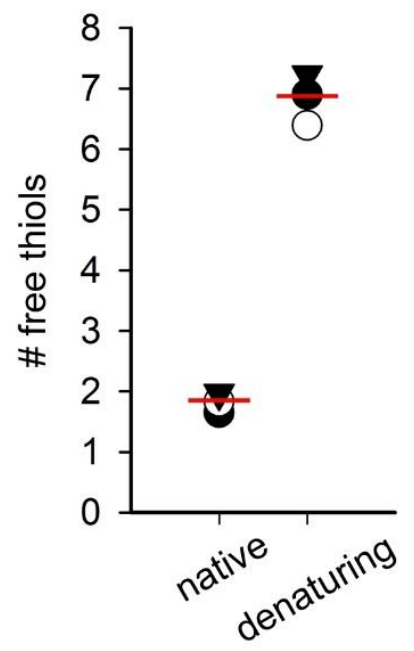

f

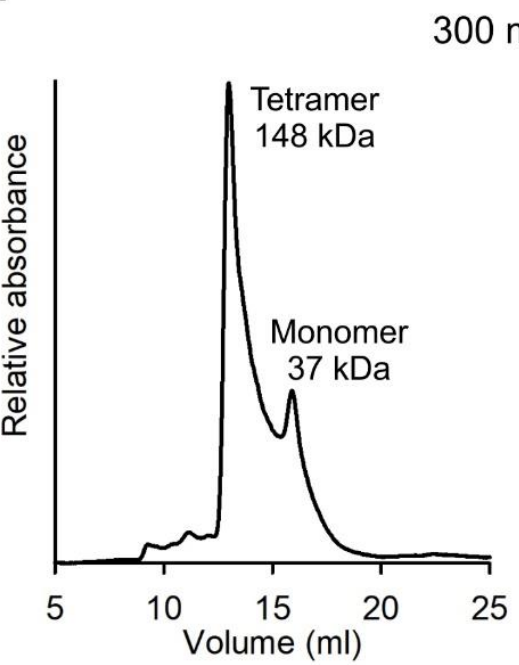

$300 \mathrm{mM} \mathrm{NaCl}$

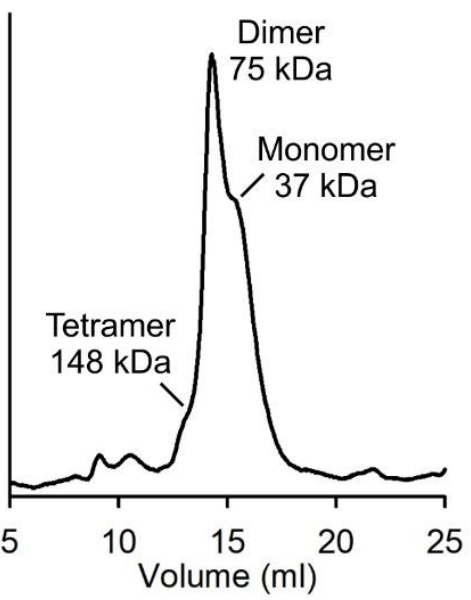

$0 \mathrm{mM} \mathrm{NaCl}$

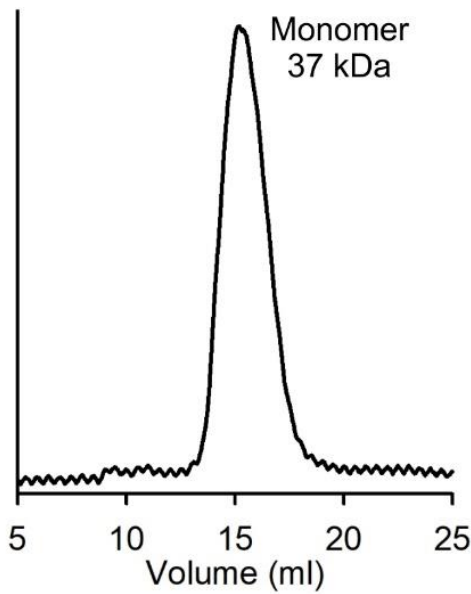




\section{Supplementary Figure 5 | Purification and characterization of recombinant His:-}

PfKelch13. (a) Construct used for the production of $\mathrm{MH}_{8} \mathrm{SR}$-tagged PfKelch13337-726 in Sf21 cells based on the released unpublished crystal structure (PDB entry 4YY8). Purification of recombinant PfKelch13 $1337-726$ by Ni-NTA affinity chromatography. Sf21 cells were lysed by three freeze-thaw cycles yielding the total cell lysate (TL). After removal of the insoluble material $(P)$, the supernatant $(L)$ was loaded on a Ni-NTA agarose column. The flow-through (FT) was discarded, the resin was washed (W) and the bound protein eluted (E) with $250 \mathrm{mM}$ imidazole. (b) Sequence coverage of purified recombinant PfKelch13337-726 by mass spectrometry. Detected peptides are highlighted in bold. (c) CD spectrum of recombinant PfKelch13 $3^{337-726}$ in $100 \mathrm{mM} \mathrm{NaF}, 50 \mathrm{mM} \mathrm{Na} \mathrm{H}_{\mathrm{y}} \mathrm{PO} 4, \mathrm{pH} 7.4$ at $20^{\circ} \mathrm{C}$. Proportions of secondary structure elements were calculated using the K2D2 software. (d) Thermal stability of recombinant PfKelch13 $3^{337-726}$ in the same buffer. (e) Detection of accessible cysteine residues of recombinant PfKelch13 $3^{337-726}$ by DTNB in the absence and presence of $2 \%$ SDS. Data and mean from three independent measurements are shown. (f) Freshly purified recombinant PfKelch13 ${ }^{337-726}$ was analyzed on a Superdex 200 Increase $10 / 300$ GL column using 50 mM $\mathrm{Na}_{x} \mathrm{H}_{\mathrm{y}} \mathrm{PO}_{4}, \mathrm{pH} 8.0$ with (left) or without (right) $300 \mathrm{mM} \mathrm{NaCl}$ as running buffer at $10^{\circ} \mathrm{C}$. The apparent molecular masses and interpreted oligomerization states are indicated. 


\section{Figure S6}

a

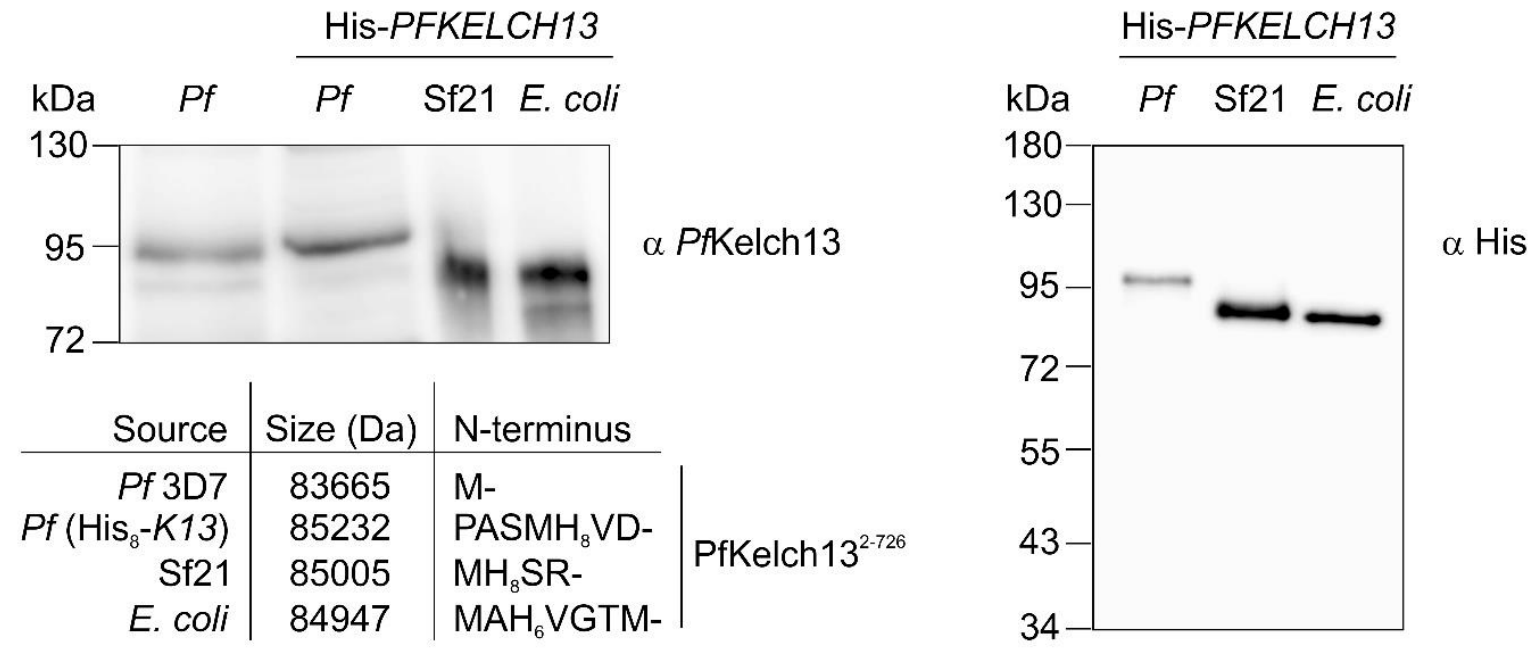

\section{b}

PASMHHHHHHHHVDEGEKVKTKANSISNFSMTYDRESGGNSNSDDKSGSSSENDSNSFMNLTSDKNEKTENN SFLLNNSSYGNVKDSLLES IDMSVLDSNFDSKKDFLPSNLSRTENNMSKDNIGNKYLNKLLNKKKDT ITNEN NN INHNNNNNNLTANN I TNNL I NNNMNS P S IMNTNKKENELDAANL INDD SGLNNLKKFSTVNNVNDTYEKK I IETELSDASDFENMVGDLRITFINWLKKTQMNFIREKDKLFKDKKELEMERVRLYKELENRKNIEEQKLHD ERKKLDIDISNGYKQI KKEKEEHRKREDEERLRFLQEIDKIKLVLYLEKEKYYQEYKNFENDKKKIVDANIA TETMIDINVGGA I EETSRHTLTQQKDSFIEKLLSGRHHVTRDKQGRIFLDRDSELFRIILNFLRNPLTIPIP KDLSESEALLKEAEFYGIKFLPFPLVFCIGGFDGVEYLNSMELLDISQQCWRMCTPMSTKKAYFGSAVLNNF LYVFGGNNYDYKALEETEVYDRLRDVWYVSSNLNI PRRNNCGVTSNGRIYCIGGYDGSS I I PNVEAYDHRMK AWVEVAPLNTPRS SAMCVAFDNKIYVIGGTNGERLNSIEVYEEKMNKWEQFPYALLEARS SGAAFNYLNQIY VVGGIDNEHNILDSVEQYQP FNKRWQFLNGVPEKKMNFGAATLSDSYIITGGENGEVLNSCHFFSPDTNEWQ LGPSLLVPRFGHSVLIANI*

\section{Supplementary Figure 6 | Potential post-translational modification of endogenous His:-}

PfKelch13. (a) N-terminally His-tagged PfKelch13 was produced in $P$. falciparum (Pf), $S$. frugiperda Sf21 cells (Sf21), and E. coli. Crude lysates were either probed with our PfKelch13 antibody from Fig. S3 (left) or an anti-His antibody (right). Crude extracts from strain 3D7 with wild-type PfKelch13 served as a control. Expected molecular masses and differences of the $\mathrm{N}$-terminal tags before residues $2-726$ are indicated. The PAS tripeptide at the $\mathrm{N}$-terminus of His-tagged PfKelch13 from P. falciparum is derived from the cleaved 2A peptide. (b) Sequence coverage of His-tagged PfKelch13 that was purified by immunoaffinity chromatography from strain His ${ }_{8}-$ PFKELCH13 ${ }^{\text {wt }}$ as shown in Fig. S3d. Detected peptides are highlighted in bold. 


\section{Figure S7}

GGTACCATGGAAGGCGAGAAGGTTAAGACCAAGGCGAATAGCATCAGCAACTTTAGCATGACCTACGA CCGTGAGAGCGGCGGCAACAGCAACAGCGACGATAAGAGCGGTAGCAGCAGCGAGAACGATAGCAACA GCTTCATGAACCTGACCAGCGACAAGAACGAAAAAACCGAGAACAACAGCTTTCTGCTGAACAACAGC AgCTACGGCAACGTGAAAGATAGCCTGCTGGAAAGCATTGACATGAGCGTTCTGGACAGCAACTTCGA TAGCAAGAAAGACTTTCTGCCGAGCAACCTGAGCCGTACCTTCAACAACATGAGCAAGGATAACATCG GTAACAAGTACCTGAACAAACTGCTGAACAAGAAAAAGGACACCATTACCAACGAGAACAACAACATC AACCACAACAACAACAACAACAACCTGACCGCGAACAACATCACCAACAACCTGATTAACAACAACAT GAACAGCCCGAGCATTATGAACACCAACAAAAAGGAAAACTTCCTGGACGCGGCGAACCTGATCAACG ACGATAGCGGTCTGAACAACCTGAAAAAGTTTAGCACCGTGAACAACGTTAACGATACCTACGAGAAG AAGATCATTGAAACCGAACTGAGCGACGCGAGCGATTTCGAGAACATGGTGGGCGACCTGCGTATCAC CTTTATTAACTGGCTGAAAAAGACCCAGATGAACTTCATCCGTGAGAAGGATAAACTGTTCAAGGACA AAAAGgAGCTGGAAATGGAGGTGTTCGTCTGTACAAGGAACTGGAGAACCGTAAAAACATTGAGGAA CAGAAGCTGCACGACGAACGTAAAAAGCTGGACATCGATATCAGCAACGGTTACAAGCAAATCAAAAA GGAGAAAGAGGAGCACCGTAAACGTTTCGATGAGGAACGTCTGCGTTTTCTGCAGGAAATCGACAAGA TTAAACTGGTGCTGTACCTGGAAAAgGAgAAGTACTACCAAGAATACAAGAACTTCGAGAACGATAAA AAGAAAATCGTGGACGCGAACATTGCGACCGAAACCATGATCGATATTAACGTTGGTGGCGCGATTTT CGAAACCAGCCGTCACACCCTGACCCAGCAAAAAGATAGCTTTATCGAGAAGCTGCTGAGCGGTCGTC ACCACGTTACCCGTGACAAACAGGGCCGTATCTTCCTGGACCGTGATAGCGAACTGTTCCGTATCATT CTGAACTTTCTGCGTAACCCGCTGACCATCCCGATTCCGAAGGACCTGAGCGAAAGCGAGGCGCTGCT GAAAGAAGCGGAGTTTTACGGTATTAAGTTCCTGCCGTTTCCGCTGGTGTTCTGCATCGGTGGCTTTG ATGGCGTTGAATATCTGAACAGCATGGAGCTGCTGGACATTAGCCAGCAATGCTGGCGTATGTGCACC CCGATGAGCACCAAGAAAGCGTACTTCGGTAGCGCGGTGCTGAACAACTTCCTGTATGTTTTTGGTGG CAACAACTACGATTATAAGGCGCTGTTTGAAACCGAGGTGTACGATCGTCTGCGTGACGTGTGGTATG TTAGCAGCAACCTGAACATCCCGCGTCGTAACAACTGCGGTGTGACCAGCAACGGCCGTATCTACTGC ATTGGTGGCTATGATGGCAGCAGCATCATTCCGAACGTTGAAGCGTACGACCACCGTATGAAAGCGTG GGTTGAGGTGGCGCCGCTGAACACCCCGCGTAGCAGCGCGATGTGCGTGGCGTTTGACAACAAGATCT ATGTTATTGGTGGCACCAACGGTGAACGTCTGAACAGCATCGAGGTGTACGAGGAAAAGATGAACAAA TGGGAACAGTTCCCGTATGCGCTGCTGGAGGCGCGTAGCAGCGGTGCGGCGTTTAACTACCTGAACCA AATCTATGTGgTTGgTGGCATTGATAACGAACACAACATCCTGGACAGCGTGGAGCAGTACCAACCGT TCAACAAACGTTGGCAGTTTCTGAACGGTGTTCCGGAGAAGAAAATGAACTTTGGCGCGGCGACCCTG AGCGATAGCTATATCATTACCGGTGGCGAAAACGGTGAGGTTCTGAACAGCTGCCACTTCTTTAGCCC GGACACCAACGAATGGCAACTGGGTCCGAGCCTGCTGGTTCCGCGTTTTGGTCATAGCGTTCTGATTG CGAATATCTAACCTAGG

\section{Supplementary Figure 7 | Sequence of E. coli codon-optimized PFKELCH13. The Kpnl} and Avrl restriction sites of pET45b are highlighted in red. 
Supplementary Table S1. Tested conditions to produce recombinant PfKelch13 and PfKelch13 $337-726$ in E. coli.

\begin{tabular}{|c|c|c|c|c|c|c|}
\hline Plasmid & Strain & Temperature & $\begin{array}{l}O_{600} \text { at } \\
\text { induction }\end{array}$ & [IPTG] & $\begin{array}{l}\text { Time of } \\
\text { expression }\end{array}$ & $\begin{array}{l}\text { Modified } \\
\text { expression }\end{array}$ \\
\hline \multirow{7}{*}{ pET45b-PFKELCH13 } & \multirow{3}{*}{$\begin{array}{l}\text { T7 SHuffle } \\
\text { Express }\end{array}$} & $30^{\circ} \mathrm{C}$ & 0.5 & $0.5 \mathrm{mM}$ & $4 \mathrm{~h}$ & \\
\hline & & $16^{\circ} \mathrm{C}$ & 0.5 & $0.2 \mathrm{mM}$ & $\mathrm{o} / \mathrm{n}$ & \\
\hline & & $30^{\circ} \mathrm{C}$ & & & $24 \mathrm{~h}$ & $\begin{array}{l}\text { Auto } \\
\text { induction }\end{array}$ \\
\hline & \multirow{2}{*}{$\begin{array}{l}\text { Rosetta- } \\
\text { gamiB(DE)pLysS }\end{array}$} & $37^{\circ} \mathrm{C}$ & 0.5 & $0.5 \mathrm{mM}$ & $4 \mathrm{~h}$ & \\
\hline & & $16^{\circ} \mathrm{C}$ & 0.5 & $0.2 \mathrm{mM}$ & $\mathrm{o} / \mathrm{n}$ & \\
\hline & \multirow{2}{*}{ BL21pLysS } & $37^{\circ} \mathrm{C}$ & 0.5 & $0.5 \mathrm{mM}$ & $4 \mathrm{~h}$ & \\
\hline & & $16^{\circ} \mathrm{C}$ & 0.5 & $0.2 \mathrm{mM}$ & $o / n$ & \\
\hline \multirow{6}{*}{$\begin{array}{l}\mathrm{pET} 45 \mathrm{~b}-P F K E L C H 13^{337-} \\
726\end{array}$} & \multirow{2}{*}{$\begin{array}{l}\text { T7 SHuffle } \\
\text { Express }\end{array}$} & $30^{\circ} \mathrm{C}$ & 0.5 & $0.5 \mathrm{mM}$ & $4 \mathrm{~h}$ & pGroEL/S \\
\hline & & $16^{\circ} \mathrm{C}$ & 0.5 & $0.2 \mathrm{mM}$ & $\mathrm{o} / \mathrm{n}$ & pGroEL/S \\
\hline & \multirow{2}{*}{$\begin{array}{l}\text { Rosetta- } \\
\text { gamiB(DE)pLysS }\end{array}$} & $37^{\circ} \mathrm{C}$ & 0.5 & $0.5 \mathrm{mM}$ & $4 \mathrm{~h}$ & \\
\hline & & $16^{\circ} \mathrm{C}$ & 0.5 & $0.2 \mathrm{mM}$ & $o / n$ & \\
\hline & \multirow{2}{*}{ BL21pLysS } & $37^{\circ} \mathrm{C}$ & 0.5 & $0.5 \mathrm{mM}$ & $4 \mathrm{~h}$ & \\
\hline & & $16^{\circ} \mathrm{C}$ & 0.5 & $0.2 \mathrm{mM}$ & $\mathrm{o} / \mathrm{n}$ & \\
\hline $\begin{array}{l}\text { pET45b- } \\
\text { PFKELCH13337-726/C4475 }\end{array}$ & $\begin{array}{l}\text { T7 SHuffle } \\
\text { Express }\end{array}$ & $16^{\circ} \mathrm{C}$ & 0.5 & $0.2 \mathrm{mM}$ & $\mathrm{o} / \mathrm{n}$ & \\
\hline $\begin{array}{l}\text { pET45b- } \\
\text { PFKELCH13337-726/C469s }\end{array}$ & $\begin{array}{l}\text { T7 SHuffle } \\
\text { Express }\end{array}$ & $16^{\circ} \mathrm{C}$ & 0.5 & $0.2 \mathrm{mM}$ & $\mathrm{o} / \mathrm{n}$ & \\
\hline $\begin{array}{l}\text { pET45b- } \\
\text { PFKELCH13337-726/C473S }\end{array}$ & $\begin{array}{l}\text { T7 SHuffle } \\
\text { Express }\end{array}$ & $16^{\circ} \mathrm{C}$ & 0.5 & $0.2 \mathrm{mM}$ & $\mathrm{o} / \mathrm{n}$ & \\
\hline $\begin{array}{l}\text { pET45b- } \\
\text { PFKELCH13337-726/C532S }\end{array}$ & $\begin{array}{l}\text { T7 SHuffle } \\
\text { Express }\end{array}$ & $16^{\circ} \mathrm{C}$ & 0.5 & $0.2 \mathrm{mM}$ & $o / n$ & \\
\hline $\begin{array}{l}\text { pET45b- } \\
\text { PFKELCH13337-726/C542S }\end{array}$ & $\begin{array}{l}\text { T7 SHuffle } \\
\text { Express }\end{array}$ & $16^{\circ} \mathrm{C}$ & 0.5 & $0.2 \mathrm{mM}$ & $\mathrm{o} / \mathrm{n}$ & \\
\hline $\begin{array}{l}\text { pET45b- } \\
\text { PFKELCH13337-726/C580s }\end{array}$ & $\begin{array}{l}\text { T7 SHuffle } \\
\text { Express }\end{array}$ & $16^{\circ} \mathrm{C}$ & 0.5 & $0.2 \mathrm{mM}$ & $\mathrm{o} / \mathrm{n}$ & \\
\hline $\begin{array}{l}\text { pET45b- } \\
\text { PFKELCH13337-726/C580Y }\end{array}$ & $\begin{array}{l}\text { T7 SHuffle } \\
\text { Express }\end{array}$ & $16^{\circ} \mathrm{C}$ & 0.5 & $0.2 \mathrm{mM}$ & $\mathrm{o} / \mathrm{n}$ & \\
\hline $\begin{array}{l}\text { pET45b- } \\
\text { PFKELCH13337-726/C696s }\end{array}$ & $\begin{array}{l}\text { T7 SHuffle } \\
\text { Express }\end{array}$ & $16^{\circ} \mathrm{C}$ & 0.5 & $0.2 \mathrm{mM}$ & $\mathrm{o} / \mathrm{n}$ & \\
\hline \multirow{5}{*}{ pQE30-PFKELCH13 } & \multirow{5}{*}{$\begin{array}{l}\text { T7 SHuffle } \\
\text { Express }\end{array}$} & \multirow{2}{*}{$30^{\circ} \mathrm{C}$} & 0.5 & \multirow{2}{*}{$0.5 \mathrm{mM}$} & \multirow{2}{*}{$4 \mathrm{~h}$} & \\
\hline & & & 1.5 & & & \\
\hline & & \multirow{3}{*}{$16^{\circ} \mathrm{C}$} & 0.5 & \multirow{3}{*}{$0.1 \mathrm{mM}$} & \multirow{3}{*}{$\mathrm{o} / \mathrm{n}$} & \\
\hline & & & 0.1 & & & \\
\hline & & & 0.1 & & & $3 \%$ ethanol \\
\hline \multirow{5}{*}{$\begin{array}{l}\mathrm{pQE} 30-P F K E L C H 13^{337-} \\
726\end{array}$} & \multirow{5}{*}{$\begin{array}{l}\text { T7 SHuffle } \\
\text { Express }\end{array}$} & \multirow{2}{*}{$30^{\circ} \mathrm{C}$} & 0.5 & \multirow{2}{*}{$0.5 \mathrm{mM}$} & \multirow{2}{*}{$4 \mathrm{~h}$} & \\
\hline & & & 1.5 & & & \\
\hline & & \multirow{3}{*}{$16^{\circ} \mathrm{C}$} & 0.5 & & & \\
\hline & & & 0.1 & $0.1 \mathrm{mM}$ & $o / n$ & \\
\hline & & & 0.1 & & & $3 \%$ ethanol \\
\hline
\end{tabular}


Supplementary Table S1. Continued.

\begin{tabular}{|c|c|c|c|c|c|c|}
\hline Plasmid & Strain & Temperature & $\begin{array}{l}O D_{600} \text { at } \\
\text { induction }\end{array}$ & [IPTG] & $\begin{array}{l}\text { Time of } \\
\text { expression }\end{array}$ & $\begin{array}{l}\text { Modified } \\
\text { expression }\end{array}$ \\
\hline \multirow{4}{*}{ pGEX-4T-1-PFKELCH13 } & \multirow{2}{*}{$\begin{array}{l}\text { T7 SHuffle } \\
\text { Express }\end{array}$} & $30^{\circ} \mathrm{C}$ & 0.5 & $0.5 \mathrm{mM}$ & $4 \mathrm{~h}$ & \\
\hline & & $16^{\circ} \mathrm{C}$ & 0.5 & $0.2 \mathrm{mM}$ & $\mathrm{o} / \mathrm{n}$ & \\
\hline & \multirow{2}{*}{ XI1blue } & $37^{\circ} \mathrm{C}$ & 0.5 & $0.5 \mathrm{mM}$ & $4 \mathrm{~h}$ & \\
\hline & & $16^{\circ} \mathrm{C}$ & 0.5 & $0.2 \mathrm{mM}$ & $\mathrm{o} / \mathrm{n}$ & \\
\hline \multirow{4}{*}{$\begin{array}{l}\text { pGEX-4T-1- } \\
\text { PFKELCH13337-726 }\end{array}$} & \multirow{2}{*}{$\begin{array}{l}\text { T7 SHuffle } \\
\text { Express }\end{array}$} & $30^{\circ} \mathrm{C}$ & 0.5 & $0.5 \mathrm{mM}$ & $4 \mathrm{~h}$ & \\
\hline & & $16^{\circ} \mathrm{C}$ & 0.5 & $0.2 \mathrm{mM}$ & $\mathrm{o} / \mathrm{n}$ & \\
\hline & \multirow{2}{*}{ XI1blue } & $37^{\circ} \mathrm{C}$ & 0.5 & $0.5 \mathrm{mM}$ & $4 \mathrm{~h}$ & \\
\hline & & $16^{\circ} \mathrm{C}$ & 0.5 & $0.2 \mathrm{mM}$ & $\mathrm{o} / \mathrm{n}$ & \\
\hline \multirow{4}{*}{$\begin{array}{l}\text { pMAL-c5x-TEV- } \\
\text { PFKELCH13 }\end{array}$} & \multirow{2}{*}{$\begin{array}{l}\text { T7 SHuffle } \\
\text { Express }\end{array}$} & $30^{\circ} \mathrm{C}$ & 0.5 & $0.5 \mathrm{mM}$ & $4 \mathrm{~h}$ & \\
\hline & & $16^{\circ} \mathrm{C}$ & 0.5 & $0.2 \mathrm{mM}$ & $\mathrm{o} / \mathrm{n}$ & \\
\hline & \multirow{2}{*}{ XI1blue } & $37^{\circ} \mathrm{C}$ & 0.5 & $0.5 \mathrm{mM}$ & $4 \mathrm{~h}$ & \\
\hline & & $16^{\circ} \mathrm{C}$ & 0.5 & $0.2 \mathrm{mM}$ & $\mathrm{o} / \mathrm{n}$ & \\
\hline \multirow{4}{*}{$\begin{array}{l}\text { pMAL-c5x-TEV- } \\
\text { PFKELCH13337-726 }\end{array}$} & \multirow{2}{*}{$\begin{array}{l}\text { T7 SHuffle } \\
\text { Express }\end{array}$} & $30^{\circ} \mathrm{C}$ & 0.5 & $0.5 \mathrm{mM}$ & $4 \mathrm{~h}$ & pGroEL/S \\
\hline & & $16^{\circ} \mathrm{C}$ & 0.5 & $0.2 \mathrm{mM}$ & $\mathrm{o} / \mathrm{n}$ & \\
\hline & \multirow{2}{*}{ XI1blue } & $37^{\circ} \mathrm{C}$ & 0.5 & $0.5 \mathrm{mM}$ & $4 \mathrm{~h}$ & \\
\hline & & $16^{\circ} \mathrm{C}$ & 0.5 & $0.2 \mathrm{mM}$ & $\mathrm{o} / \mathrm{n}$ & \\
\hline
\end{tabular}


Supplementary Table S2. List of primers used for cloning of SLI and expression constructs. Restriction sites are underlined.

\begin{tabular}{|c|c|c|}
\hline Number & Name & Sequence $\left(5^{\prime} \rightarrow 3^{\prime}\right)$ \\
\hline P1 & PfK13/337-726/Kpnl/s & GATCGGTACCGATAAAAAGAAAATCGTGGACGCG \\
\hline P2 & PfK13/Avrll/as & GATCCCTAGGTTAGATATTCGCAATCAGAA \\
\hline P3 & PfK13/BamHI/s & GATCGGATCCATGGAAGGCGAGAAGGTTAAGACC \\
\hline P4 & PfK13/HindIII/as & GATCAAGCTTTTAGATATTCGCAATCAGAACGCTATG \\
\hline P5 & PfK13/BamHI/337-726/s & GATCGGATCCGATAAAAAGAAAATCGTGGACGCGAAC \\
\hline P26 & $\mathrm{PfK} 13 / \mathrm{Xbal} / 337-726 / \mathrm{s}$ & GATCTCTAGAGATAAAAAGAAAATCGTGGACGCGAAC \\
\hline P27 & $\mathrm{PfK} 13 / \mathrm{Xbal} / \mathrm{s}$ & GATCTCTAGAATGGAAGGCGAGAAGGTTAAGACC \\
\hline P28 & BgIII/ATG6xHis/Kpnl/Ncol/s & GATCTATGCACCATCATCACCATCACGGTACCC \\
\hline P29 & Bglll/ATG6xHis/Kpnl/Ncol/as & CATGGGGTACCGTGATGGTGATGATGGTGCATA \\
\hline P30 & PfK13/Notl/as & GATCGCGGCCGCTTAGATATTCGCAATCAGAACGCTATG \\
\hline P31 & $\mathrm{PfK} 13 / \mathrm{Kpnl} / \mathrm{s}$ & GATCGGTACCATGGAAGGCGAGAAGG \\
\hline P32 & $\mathrm{PfK} 13 / \mathrm{Ndel} / \mathrm{s}$ & GATCCATATGGAAGGCGAGAAGGTTAAGACC \\
\hline P33 & PfK13/337-726/Ndel/s & GATCCATATGGATAAAAAGAAAATCGTGGACGCG \\
\hline P38 & PfK13_Xbal_N8Hisup_s & GATCTCTAGACCACCACCACCATCACCACCATCACATGGAAGGCGAGAAGG \\
\hline P39 & PfK13_EcoRI_N8Hisup_s & GATCGAATTCACCACCACCATCACCACCATCACATGGAAGGCGAGAAGG \\
\hline P50 & BamHI/ATG8xHis/Xbal/s & GATCCATGCATCATCATCATCATCATCATCATI \\
\hline P51 & BamHI/ATG8xHis/Xbal/as & 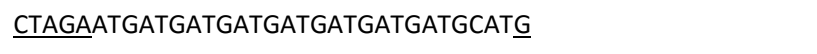 \\
\hline P52 & $\mathrm{PfK} 13 / \mathrm{Xbal} / \mathrm{s}$ & GATCTCTAGAGAAGGCGAGAAGGTTAAG \\
\hline P60 & Nhel/ATG8xHis/Sall/s & $\underline{\text { CTAGCATGCATCATCATCATCATCATCATCATG }}$ \\
\hline P61 & Nhel/ATG8xHis/Sall/as & $\underline{\text { TCGACATGATGATGATGATGATGATGATGCATG }}$ \\
\hline P62 & BgllI/L3rest/Sall/s & $\underline{\text { GATCTGCTGCTGCTGGTGCTGGTGGTGCTGCTAGAGCTGCTG }}$ \\
\hline P63 & BgIII/L3rest/Sall/as & $\underline{\text { TCGACAGCAGCTCTAGCAGCACCACCAGCACCAGCAGCAGCA }}$ \\
\hline P64 & pSLI-PfK13 $3^{337-726} /$ Sall/s & GATCGTCGACGACAAGAAGAAGATAGTAGACGC \\
\hline P65 & pSLI-PfK13 ${ }^{337-726} /$ oxP/Xhol/as & GATCCTCGAGTCATAATAACTTCGTATAATG \\
\hline P69 & pSLI-K13/Sall/s & GATCGTCGACGAGGGTGAGAAGGTTAAGACTAAAG \\
\hline P88 & Stul_glmS_s & GATCAGGCCTGTCCAGACCTGCAG \\
\hline P89 & glmS_Xhol_as & GATCCTCGAGAGATCATGTGATTTCTCTTTG \\
\hline P90 & GFP/spel/s & GATCACTAGTATGAGTAAAGGAGAAGAACTTTTCACTG \\
\hline P91 & L3/BamHI/as & GATCGGATCCAGCAGCTCTAGCAGCACC \\
\hline P92 & $\mathrm{K} 13 / \mathrm{Sacl} / \mathrm{as}$ & GATCGAGCTCTTAGATATTCGCAATCAGAACGC \\
\hline
\end{tabular}


Supplementary Table S3. List of mutagenesis primers. Mutations are indicated in red.

\begin{tabular}{|c|c|c|}
\hline Number & Name & Sequence $\left(5^{\prime} \rightarrow 3^{\prime}\right)$ \\
\hline P6 & $\mathrm{PfK} 13 / \mathrm{C} 447 \mathrm{~S} / \mathrm{s}$ & GTTTCCGCTGGTGTTCAGCATCGGTGGCTTTG \\
\hline P7 & PfK13/C447S/as & CAAAGCCACCGATGCTGAACACCAGCGGAAAC \\
\hline P8 & PfK13/C469S/s & GACATTAGCCAGCAAAGCTGGCGTATGTGCAC \\
\hline P9 & PfK13/C469S/as & GTGCACATACGCCAGCTTTGCTGGCTAATGTC \\
\hline P10 & PfK13/C473S/s & CAATGCTGGCGTATGAGCACCCCGATGAGC \\
\hline P11 & PfK13/C473S/as & GCTCATCGGGGTGCTCATACGCCAGCATTG \\
\hline P12 & PfK13/C532S/s & CGCGTCGTAACAACAGCGGTGTGACCAGCAAC \\
\hline P13 & PfK13/C532S/as & GTTGCTGGTCACACCGCTGTTGTTACGACGCG \\
\hline P14 & PfK13/C542S/s & CAACGGCCGTATCTACAGCATTGGTGGCTATG \\
\hline P15 & PfK13/C542S/as & CATAGCCACCAATGCTGTAGATACGGCCGTTG \\
\hline P16 & PfK13/C580S/s & GTAGCAGCGCGATGAGCGTGGCGTTTGACAAC \\
\hline P17 & PfK13/C580S/as & GTTGTCAAACGCCACGCTCATCGCGCTGCTAC \\
\hline P18 & PfK13/C696S/s & GAGGTTCTGAACAGCAGCCACTTCTTTAGCC \\
\hline P19 & PfK13/C696S/as & GGCTAAAGAAGTGGCTGCTGTTCAGAACCTC \\
\hline P20 & PfK13/C580F/s & GTAGCAGCGCGATGTTCGTGGCGTTTGACAAC \\
\hline P21 & PfK13/C580F/as & GTTGTCAAACGCCACGAACATCGCGCTGCTAC \\
\hline P22 & PfK13/C580Y/s & GCGTAGCAGCGCGATGTATGTGGCGTTTGACAACAAG \\
\hline P23 & PfK13/C580Y/as & CTTGTTGTCAAACGCCACATACATCGCGCTGCTACGC \\
\hline P71 & $\mathrm{pSLI}-\mathrm{K} 13 \mathrm{C} 580 \mathrm{~S} / \mathrm{s}$ & GGTCTAGTGCAATGTCCGTAGCATTCGACAAC \\
\hline P72 & pSLI-K13C580S/as & GTTGTCGAATGCTACGGACATTGCACTAGACC \\
\hline P73 & pSLI-K13C580Y/s & GGTCTAGTGCAATGTACGTAGCATTCGACAAC \\
\hline P74 & pSLI-K13C580Y/as & GTTGTCGAATGCTACGTACATTGCACTAGACC \\
\hline P75 & $\mathrm{pSLI}-\mathrm{K} 13 \mathrm{C} 532 \mathrm{~S} / \mathrm{s}$ & CAAGGAGGAACAACTCCGGAGTAACCTCTAAC \\
\hline P76 & pSLI-K13C532S/as & GTTAGAGGTTACTCCGGAGTTGTTCCTCCTTG \\
\hline P84 & pSLI-K13C469S/s & GACATATCACAGCAGAGTTGGCGAATGTGC \\
\hline P85 & pSLI-K13C469S/as & GCACATTCGCCAACTCTGCTGTGATATGTC \\
\hline P86 & $\mathrm{pSLI}-\mathrm{K} 13 \mathrm{C} 473 \mathrm{~S} / \mathrm{s}$ & CAGCAGTGTTGGCGAATGAGTACTCCAATGTCAACTAAG \\
\hline P87 & pSLI-K13C473S/as & CTTAGTTGACATTGGAGTACTCATTCGCCAACACTGCTG \\
\hline P95 & PfK13/R539T/s & GTGACCAGCAACGGCACTATCTACTGCATTG \\
\hline P96 & PfK13/R539T/as & CAATGCAGTAGATAGTGCCGTTGCTGGTCAC \\
\hline P97 & PfK13/I543T/s & CCGTATCTACTGCACTGGTGGCTATGATG \\
\hline P98 & PfK13/I543T/as & CATCATAGCCACCAGTGCAGTAGATACGG \\
\hline
\end{tabular}


Supplementary Table S4. List of sequencing and genotyping primers.

\begin{tabular}{lll} 
Number & Name & Sequence $\left(\mathbf{5}^{\prime} \rightarrow \mathbf{3}^{\prime}\right)$ \\
Sequencing primers & \\
\hline P24 & PfK13/sequencing1/s & CCTGGACGCGGCGAACC \\
P25 & PfK13/sequencing2/as & GCTGCCATCATAGCCACC \\
P40 & T7promoter & TAATACGACTCACTATAGGG \\
P53 & pUC/M13 Forward & CCCAGTCACGACGTTGTAAAACG \\
P54 & pUC/M13 Reverse & AGCGGATAACAATTTCACACAGG \\
P55 & pVL1392.seqF & AAATGATAACCATCTCGC \\
P66 & pSLI-N-loxP/s & GTTCTTTGTTGACTTGTG \\
P67 & pSLI-N-loxP/as & CAGTTATAAATACAATCAATTGG \\
P68 & GFPseq.end/s & GTAACAGCTGCTGG \\
P70 & pSLI-K13/s & CAAGGACAAAAAGGAGC \\
P83 & K13-SLI/seq/s & CTCACCTTCTATAATGAACAC \\
P93 & pHBIRH/seq/s & CCTACATACATATACAAACCTAC \\
P94 & pHBIRH/seq/as & CATATGTATTTTTTTGTAATTTCTGTG
\end{tabular}

Genotyping primers (PfKelch13 SLI)

\begin{tabular}{lll}
\hline P79 & K13_int_check_5/s & CAATTATGAATACCAACAAAAAAGAG \\
P80 & K13_int_check_3/as & GTTTCAAAAATAGCTCCACC \\
P81 & pARL sense 55 & GAGCGGATAACAATTTCAC \\
P82 & yDHODH/as & GAGTGTAAAATGCACGAAC
\end{tabular}

